\title{
Stability of the 1D IBVP for a Non Autonomous Scalar Conservation Law
}

\author{
Rinaldo M. Colombo* Elena Rossi ${ }^{\dagger}$
}

INDAM Unit, University of Brescia, Italy

\begin{abstract}
We prove the stability with respect to the flux of solutions to initial - boundary value problems for scalar non autonomous conservation laws in one space dimension. Key estimates are obtained through a careful construction of the solutions.

2010 Mathematics Subject Classification: 35L65, 35L04

Keywords: Conservation Laws, Boundary Value Problems for Conservation Laws
\end{abstract}

\section{Introduction}

This paper deals with the Initial Boundary Value Problem (IBVP) for a possibly non autonomous scalar conservation law on a half-line

$$
\left\{\begin{array}{lc}
\partial_{t} u+\partial_{x} f(t, u)=0 & (t, x) \in[0, T] \times \mathbb{R}_{+} \\
u(0, x)=u_{o}(x) & x \in \mathbb{R}_{+} \\
u(t, 0)=u_{b}(t) & t \in[0, T],
\end{array}\right.
$$

or on a segment

$$
\left\{\begin{array}{lc}
\partial_{t} u+\partial_{x} f(t, u)=0 & (t, x) \in[0, T] \times[0, L] \\
u(0, x)=u_{o}(x) & x \in[0, L] \\
u(t, 0)=u_{b, 1}(t) & t \in[0, T] \\
u(t, L)=u_{b, 2}(t) & t \in[0, T] .
\end{array}\right.
$$

For these problems, we complete the basic well posedness and stability results. That is, we detail below the proofs of the existence of solutions and of their stability with respect to the flow. For the Lipschitz continuous dependence of solutions on initial and boundary data we refer to [4, 8].

With a slight abuse of notation, we refer to the non autonomous (time dependent), respectively autonomous (time independent), case as to the case where the flux $f$ depends explicitly on time $t$ or not. In both cases, boundary data are time dependent.

${ }^{*}$ rinaldo.colombo@unibs.it

†elena.rossi@unibs.it 
Conservation Laws are typically studied either in the case of one dimensional systems or of scalar multi-dimensional equations. In the former case, we refer to [1, 10, 12] for the basic existence results and for discussions on the very definition of solution to the initial boundary value problem. Differently from these works, the present paper deals with the stability with respect to the flow and covers also the case of a time dependent flow.

In the scalar multi-dimensional case, the key reference is [4], see also [8, 9, 15, 16, 17, [18, 19, 20], which considers the existence of solutions and their continuous dependence on initial and boundary data but only on bounded domains. Here, in addition, we deal also with unbounded domains and ensure the stability with respect to the flow, though limited to the one dimensional case. We stress here the key role played by the definition of solutions to (1.1) or 1.2) as provided in [16, 20]. Indeed, this definition is stable under $\mathbf{L}^{1}$-convergence, see [15, Chapter 2, Remark 7.33], and its use allows to avoid all issues related to the limit of traces converging to the trace of the limit.

Recall that in the case of the autonomous Cauchy problem, the stability of solutions with respect to the flux is treated in [13, Theorem 2.13]. In one space dimension, [5, Theorem 2.6] deals with a convex scalar time independent flux, while autonomous systems are considered in [5, Theorem 2.1]. Here, we extend these results to the non autonomous case with boundary, albeit in the scalar one dimensional case.

A key role in this paper is played by the wave front tracking technique, see [6, 9]. In this framework, Glimm type functionals yield a precise control of the total variation. As a consequence, we obtain the stability of solutions with respect to the flux in the autonomous case, thanks to a careful use of [6. Theorem 2.9]. All these estimates then lead to the stability in the time dependent case.

The next section presents the results concerning (1.1) on a half line. Section 3 deals with 1.2 on a segment. In both cases, we present first the autonomous case and then the non autonomous one. Section 4 is devoted to proofs.

\section{The Case of the Half-Line}

All statements and proofs below are referred to the time interval $[0, T]$ for a fixed $T>0$. Where the extension to $t \in \mathbb{R}_{+}$is not straightforward, we provide all necessary details. Denote $\mathbb{R}_{+}=\left[0,+\infty\left[\right.\right.$ and $\left.\stackrel{\circ}{R}_{+}=\right] 0,+\infty[$. Following [16, 20], for $a, b \in \mathbb{R}$, we let

$$
\mathcal{I}(a, b)=[\min \{a, b\}, \max \{a, b\}] .
$$

Below, if $u_{\ell} \in \mathbf{L}^{\infty}\left(I_{\ell} ; \mathbb{R}\right)$ for real intervals $I_{\ell}$ and for $\ell=1, \ldots, m$, we define

$$
\mathcal{U}\left(u_{1}, \ldots, u_{m}\right)=\left[\min _{\ell=1, \ldots, m} \underset{I_{\ell}}{\operatorname{essinf}} u_{\ell}, \underset{\ell=1, \ldots, m}{\max } \operatorname{ess\operatorname {sup}} u_{\ell}\right] .
$$

Equivalently, $\mathcal{U}\left(u_{1}, \ldots, u_{m}\right)$ is the closed convex hull of $\bigcup_{\ell=1}^{m} u_{\ell}\left(I_{\ell}\right)$. If $I_{u}$ is a real interval, for $u \in \mathbf{B V}\left(I_{u} ; \mathbb{R}\right), \mathrm{TV}(u)$ stands for the total variation of $u$ on $I_{u}$, see [11, §5.10.1] and, for any interval $I \subseteq I_{u}$, we also set TV $(u ; I)=\mathrm{TV}\left(u_{\mid I}\right)$. Moreover, for $\boldsymbol{u} \in \mathbf{B V}\left(I ; \mathbb{R}^{m}\right)$, we define $\mathrm{TV}(\boldsymbol{u})=\sum_{\ell=1}^{m} \mathrm{TV}\left(u_{\ell}\right)$. Denote by $\mathscr{T}_{t}$ the $t$-translation operator:

$$
\left(\mathscr{T}_{t} u\right)(\tau)=u(t+\tau)
$$


As usual, $u(t, 0+)=\lim _{x \rightarrow 0+} u(t, x)$ stands for the trace at 0 from the right, see [11, Paragraph 5.3] or [8, Appendix]. Throughout, we set

$$
\begin{aligned}
\operatorname{sgn}^{+}(u) & = \begin{cases}1 & \text { if } u>0, \\
0 & \text { if } u \leq 0,\end{cases} & \operatorname{sgn}^{-}(u) & = \begin{cases}0 & \text { if } u \geq 0, \\
-1 & \text { if } u<0,\end{cases} \\
u^{+} & =\max \{u, 0\}, & u^{-} & =\max \{-u, 0\} .
\end{aligned}
$$

Introduce the semi-Kružkov entropy-entropy flux pairs, see [16, 20]: for any $k \in \mathbb{R}$

$$
\begin{array}{ll}
\eta_{k}^{+}(u)=(u-k)^{+}, & \Phi_{k}^{+}(t, u)=\operatorname{sgn}^{+}(u-k)(f(t, u)-f(t, k)), \\
\eta_{k}^{-}(u)=(u-k)^{-}, & \Phi_{k}^{-}(t, u)=\operatorname{sgn}^{-}(u-k)(f(t, u)-f(t, k)) .
\end{array}
$$

Definition 2.1. A weak entropy solution to the IBVP (1.1) on the interval $[0, T]$ is a map $u \in \mathbf{L}^{\infty}\left([0, T] \times \mathbb{R}_{+} ; \mathbb{R}\right)$ such that for any $k \in \mathbb{R}$ and for any test function $\varphi \in \mathbf{C}_{c}^{1}\left(\mathbb{R} \times \mathbb{R} ; \mathbb{R}_{+}\right)$

$$
\begin{aligned}
& \int_{0}^{T} \int_{\mathbb{R}_{+}}\left\{\eta_{k}^{+}(u(t, x)) \partial_{t} \varphi(t, x)+\Phi_{k}^{+}(t, u(t, x)) \partial_{x} \varphi(t, x)\right\} \mathrm{d} x \mathrm{~d} t \\
& +\int_{\mathbb{R}_{+}} \eta_{k}^{+}\left(u_{o}(x)\right) \varphi(0, x) \mathrm{d} x-\int_{\mathbb{R}_{+}} \eta_{k}^{+}(u(T, x)) \varphi(T, x) \mathrm{d} x \\
& +\left\|\partial_{u} f\right\|_{\mathbf{L}^{\infty}([0, T] \times \mathcal{U} ; \mathbb{R})} \int_{0}^{T} \eta_{k}^{+}\left(u_{b}(t)\right) \varphi(t, 0) \mathrm{d} t \geq 0,
\end{aligned}
$$

and

$$
\begin{aligned}
& \int_{0}^{T} \int_{\mathbb{R}_{+}}\left\{\eta_{k}^{-}(u(t, x)) \partial_{t} \varphi(t, x)+\Phi_{k}^{-}(t, u(t, x)) \partial_{x} \varphi(t, x)\right\} \mathrm{d} x \mathrm{~d} t \\
& +\int_{\mathbb{R}_{+}} \eta_{k}^{-}\left(u_{o}(x)\right) \varphi(0, x) \mathrm{d} x-\int_{\mathbb{R}_{+}} \eta_{k}^{-}(u(T, x)) \varphi(T, x) \mathrm{d} x \\
& +\left\|\partial_{u} f\right\|_{\mathbf{L}^{\infty}([0, T] \times \mathcal{U} ; \mathbb{R})} \int_{0}^{T} \eta_{k}^{-}\left(u_{b}(t)\right) \varphi(t, 0) \mathrm{d} t \geq 0,
\end{aligned}
$$

where $\mathcal{U}=\mathcal{U}\left(u_{o}, u_{b \mid[0, T]}\right)$ as in 2.2 .

Relying essentially solely on Definition 2.1, one obtains the Lipschitz continuous dependence of the solution to 1.1 on initial and boundary data.

Proposition 2.2. Let $f \in \mathbf{C}^{1}([0, T] \times \mathbb{R} ; \mathbb{R})$ be such that $\left\{u \mapsto \partial_{t} f(t . u)\right\} \in \mathbf{W}_{\text {loc }}^{1, \infty}(\mathbb{R} ; \mathbb{R})$ for all $t \in[0, T], u_{o}, w_{o} \in\left(\mathbf{L}^{1} \cap \mathbf{L}^{\infty}\right)\left(\mathbb{R}_{+} ; \mathbb{R}\right)$ and $u_{b}, w_{b} \in\left(\mathbf{L}^{1} \cap \mathbf{L}^{\infty}\right)([0, T] ; \mathbb{R})$. Assume the problems

$$
\left\{\begin{array} { l c } 
{ \partial _ { t } u + \partial _ { x } f ( t , u ) = 0 } & { ( t , x ) \in [ 0 , T ] \times \mathbb { R } _ { + } } \\
{ u ( 0 , x ) = u _ { o } ( x ) } & { x \in \mathbb { R } _ { + } } \\
{ u ( t , 0 + ) = u _ { b } ( t ) } & { t \in [ 0 , T ] }
\end{array} \text { and } \left\{\begin{array}{ll}
\partial_{t} w+\partial_{x} f(t, w)=0 & (t, x) \in[0, T] \times \mathbb{R}_{+} \\
w(0, x)=w_{o}(x) & x \in \mathbb{R}_{+} \\
w(t, 0+)=w_{b}(t) & t \in[0, T]
\end{array}\right.\right.
$$

admit solutions $u, w \in \mathbf{L}^{\infty}\left([0, T] \times \mathbb{R}_{+} ; \mathbb{R}\right)$ in the sense of Definition 2.1 , such that $u$ and $w$ both admit a trace for $x \rightarrow 0+$ for a.e. $t \in[0, T]$. Then, for all $t \in[0, T]$,

$$
\|u(t)-w(t)\|_{\mathbf{L}^{1}\left(\mathbb{R}_{+} ; \mathbb{R}\right)} \leq\left\|u_{o}-w_{o}\right\|_{\mathbf{L}^{1}\left(\mathbb{R}_{+} ; \mathbb{R}\right)}+\left\|\partial_{u} f\right\|_{\mathbf{L}^{\infty}([0, t] \times \mathcal{U} ; \mathbb{R})}\left\|u_{b}-w_{b}\right\|_{\mathbf{L}^{1}([0, t] ; \mathbb{R})}
$$

where $\mathcal{U}=\mathcal{U}\left(u_{b \mid[0, t]}, w_{b \mid[0, t]}\right)$ is as in $(2.2]$. 
Remark that Proposition 2.2, whose proof is deferred to Section 4 , also ensures the uniqueness of the solution to (1.1) in the sense of Definition 2.1, as soon as a solution exists.

\subsection{The Autonomous Case on the Half-Line}

We study first the following autonomous IBVP, which is a particular case of (1.1):

$$
\left\{\begin{array}{lc}
\partial_{t} u+\partial_{x} f(u)=0 & (t, x) \in[0, T] \times \mathbb{R}_{+} \\
u(0, x)=u_{o}(x) & x \in \mathbb{R}_{+} \\
u(t, 0)=u_{b}(t) & t \in[0, T] .
\end{array}\right.
$$

Solutions to (2.8) are understood in the sense of Definition 2.1. Observe that Proposition 2.2 applies to (2.8), under the hypothesis $f \in \mathbf{C}^{1}(\mathbb{R} ; \mathbb{R})$. The next Proposition ensures the existence of solutions to (2.8), as well as some of their properties.

Proposition 2.3. Let $f \in \mathbf{W}_{\text {loc }}^{1, \infty}(\mathbb{R} ; \mathbb{R}), u_{o} \in\left(\mathbf{L}^{1} \cap \mathbf{B V}\right)\left(\mathbb{R}_{+} ; \mathbb{R}\right)$ and $u_{b} \in\left(\mathbf{L}^{1} \cap \mathbf{B V}\right)([0, T] ; \mathbb{R})$. Then, problem (2.8) admits a solution $u$ in the sense of Definition 2.1. with the properties:

1. If $u_{o}$ and $u_{b}$ are piecewise constant, then for $t$ small, the map $t \rightarrow u(t)$ coincides with the gluing of Lax solutions to Riemann problems at the points of jumps of $u_{o}$ and at $x=0$.

2. Range of $u$ : with the notation in (2.2), $u(t, x) \in \mathcal{U}\left(u_{o}, u_{b \mid[0, t]}\right)$ for a.e. $(t, x) \in[0, T] \times \mathbb{R}_{+}$. Hence, for a.e. $t \in[0, T]$,

$$
\|u(t)\|_{\mathbf{L}^{\infty}\left(\mathbb{R}_{+} ; \mathbb{R}\right)} \leq \max \left\{\left\|u_{o}\right\|_{\mathbf{L}^{\infty}\left(\mathbb{R}_{+} ; \mathbb{R}\right)},\left\|u_{b}\right\|_{\mathbf{L}^{\infty}([0, t] ; \mathbb{R})}\right\}
$$

3. $u$ is Lipschitz continuous in time: for all $t_{1}, t_{2} \in[0, T]$,

$$
\left\|u\left(t_{1}\right)-u\left(t_{2}\right)\right\|_{\mathbf{L}^{1}\left(\mathbb{R}_{+} ; \mathbb{R}\right)} \leq C_{1}\left|t_{2}-t_{1}\right|
$$

where $C_{1}=\left\|f^{\prime}\right\|_{\mathbf{L}^{\infty}(\mathcal{U} ; \mathbb{R})}\left(\operatorname{TV}\left(u_{o}\right)+\operatorname{TV}\left(u_{b} ;\left[0, t_{1} \vee t_{2}\right]\right)+\left|u_{b}(0+)-u_{o}(0+)\right|\right)$ and $\mathcal{U}=$ $\mathcal{U}\left(u_{o}, u_{b \mid\left[0, t_{1} \vee t_{2}\right]}\right)$, with the notation 2.2 .

4. Total variation estimate: for all $t \in[0, T]$

$$
\operatorname{TV}(u(t)) \leq \operatorname{TV}\left(u_{o}\right)+\operatorname{TV}\left(u_{b} ;[0, t]\right)+\left|u_{b}(0+)-u_{o}(0+)\right|
$$

The proof is deferred to $\S 4.1$. Various results similar to, but not containing, Proposition 2.3 can be found in the current literature. The case of a convex flux is treated in [3]. A bounded domain is considered in [4] and in [2, 8], see also [9, Section 6.9] or [19, Section 15.1].

Our main result, namely the stability of the solution to 2.8 with respect to the flux, concludes this Section. The current literature consider the case when no boundary is present. In the one dimensional setting, the scalar equation is treated in [13, Theorem 2.13] and [5, Theorem 2.6] for a convex scalar flux, while systems are considered in [5, Theorem 2.1]. The multi-dimensional case is covered in [7]. 
Theorem 2.4. Let $f, g \in \mathbf{C}^{1}(\mathbb{R} ; \mathbb{R}), u_{o} \in\left(\mathbf{L}^{1} \cap \mathbf{B V}\right)\left(\mathbb{R}_{+} ; \mathbb{R}\right)$ and $u_{b} \in\left(\mathbf{L}^{1} \cap \mathbf{B V}\right)([0, T] ; \mathbb{R})$. Call $u$ and $v$ the solutions to the problems

$\left\{\begin{array}{lc}\partial_{t} u+\partial_{x} f(u)=0 & (t, x) \in[0, T] \times \mathbb{R}_{+} \\ u(0, x)=u_{o}(x) & x \in \mathbb{R}_{+} \\ u(t, 0)=u_{b}(t) & t \in[0, T]\end{array} \quad\right.$ and $\left\{\begin{array}{lc}\partial_{t} v+\partial_{x} g(v)=0 & (t, x) \in[0, T] \times \mathbb{R}_{+} \\ v(0, x)=u_{o}(x) & x \in \mathbb{R}_{+} \\ v(t, 0)=u_{b}(t) & t \in[0, T]\end{array}\right.$

constructed in Proposition 2.3. Then, with $\mathcal{U}=\mathcal{U}\left(u_{o}, u_{b \mid[0, t]}\right)$ as in 2.2$]$, for all $t \in[0, T]$,

$$
\begin{aligned}
\|u(t)-v(t)\|_{\mathbf{L}^{1}\left(\mathbb{R}_{+} ; \mathbb{R}\right)} \leq & \max \left\{1,\left\|g^{\prime}\right\|_{\mathbf{L}^{\infty}(\mathcal{U} ; \mathbb{R})}\right\}\|D(f-g)\|_{\mathbf{L}^{\infty}(\mathcal{U} ; \mathbb{R})} \\
& \times\left(\operatorname{TV}\left(u_{o}\right)+\operatorname{TV}\left(u_{b} ;[0, t]\right)+\left|u_{b}(0+)-u_{o}(0+)\right|\right) t .
\end{aligned}
$$

The proof is deferred to Section 4.1.

\subsection{The Non Autonomous Case on the Half-Line}

The results obtained in Section 2.1 are here extended to problem 1.1). We first generalize Proposition 2.3 .

Proposition 2.5. Let $f$ be such that

$$
f \in \mathbf{C}^{1}([0, T] \times \mathbb{R} ; \mathbb{R}) \quad \text { and } \quad\left\{t \mapsto \partial_{u} f(t, u)\right\} \in \mathbf{W}_{\text {loc }}^{1, \infty}([0, T] ; \mathbb{R}) \text { for all } u \in \mathbb{R} .
$$

Fix $u_{o} \in\left(\mathbf{L}^{1} \cap \mathbf{B V}\right)\left(\mathbb{R}_{+} ; \mathbb{R}\right)$ and $u_{b} \in\left(\mathbf{L}^{1} \cap \mathbf{B V}\right)([0, T] ; \mathbb{R})$. Then, problem (1.1) admits a solution $u$ in the sense of Definition 2.1, with the properties:

1. Range of $u$ : with the notation in $(2.2), u(t, x) \in \mathcal{U}\left(u_{o}, u_{b \mid[0, t]}\right)$ for a.e. $(t, x) \in[0, T] \times \mathbb{R}_{+}$. Hence, for a.e. $t \in[0, T]$,

$$
\|u(t)\|_{\mathbf{L}^{\infty}\left(\mathbb{R}_{+} ; \mathbb{R}\right)} \leq \max \left\{\left\|u_{o}\right\|_{\mathbf{L}^{\infty}\left(\mathbb{R}_{+} ; \mathbb{R}\right)},\left\|u_{b}\right\|_{\mathbf{L}^{\infty}([0, t] ; \mathbb{R})}\right\} .
$$

2. $u$ is Lipschitz continuous in time: for all $t_{1}, t_{2} \in[0, T]$,

$$
\left\|u\left(t_{1}\right)-u\left(t_{2}\right)\right\|_{\mathbf{L}^{1}\left(\mathbb{R}_{+} ; \mathbb{R}\right)} \leq C\left|t_{2}-t_{1}\right|,
$$

where $C=\left\|\partial_{u} f\right\|_{\mathbf{L}^{\infty}\left(\left[0, t_{1} \vee t_{2}\right] \times \mathcal{U} ; \mathbb{R}\right)}\left(\mathrm{TV}\left(u_{o}\right)+\mathrm{TV}\left(u_{b} ;\left[0, t_{1} \vee t_{2}\right]\right)+\left|u_{b}(0+)-u_{o}(0+)\right|\right)$ and $\mathcal{U}=\mathcal{U}\left(u_{o}, u_{b \mid\left[0, t_{1} \vee t_{2}\right]}\right)$, with the notation 2.2 .

3. Total variation estimate: for all $t \in[0, T]$

$$
\operatorname{TV}(u(t)) \leq \operatorname{TV}\left(u_{o}\right)+\operatorname{TV}\left(u_{b} ;[0, t]\right)+\left|u_{b}(0+)-u_{o}(0+)\right|
$$

The proof is deferred to $\S 4.2$. 
Theorem 2.6. Let $f$ and $g$ both satisfy 2.10 . Fix $u_{o} \in\left(\mathbf{L}^{1} \cap \mathbf{B V}\right)\left(\mathbb{R}_{+} ; \mathbb{R}\right)$ and $u_{b} \in$ $\left(\mathbf{L}^{1} \cap \mathbf{B V}\right)([0, T] ; \mathbb{R})$. Call $u$ and $v$ the solutions to the problems

$\left\{\begin{array}{lr}\partial_{t} u+\partial_{x} f(t, u)=0 & (t, x) \in[0, T] \times \mathbb{R}_{+} \\ u(0, x)=u_{o}(x) & x \in \mathbb{R}_{+} \\ u(t, 0)=u_{b}(t) & t \in[0, T]\end{array} \quad\right.$ and $\begin{cases}\partial_{t} v+\partial_{x} g(t, v)=0 & (t, x) \in[0, T] \times \mathbb{R}_{+} \\ v(0, x)=u_{o}(x) & x \in \mathbb{R}_{+} \\ v(t, 0)=u_{b}(t) & t \in[0, T]\end{cases}$ constructed in Proposition 2.5. Then, with $\mathcal{U}=\mathcal{U}\left(u_{o}, u_{b \mid[0, t]}\right)$ as in 2.2. for all $t \in[0, T]$

$$
\begin{aligned}
\|u(t)-v(t)\|_{\mathbf{L}^{1}\left(\mathbb{R}_{+} ; \mathbb{R}\right)} \leq & \max \left\{1,\left\|\partial_{u} g\right\|_{\mathbf{L}^{\infty}([0, t] \times \mathcal{U} ; \mathbb{R})}\right\}\left\|\partial_{u}(f-g)\right\|_{\mathbf{L}^{\infty}([0, t] \times \mathcal{U} ; \mathbb{R})} \\
& \times\left(\operatorname{TV}\left(u_{o}\right)+\operatorname{TV}\left(u_{b} ;[0, t]\right)+\left|u_{b}(0+)-u_{o}(0+)\right|\right) t .
\end{aligned}
$$

\section{The Case of the Segment}

We consider here the case 1.2 where $x$ varies in a segment. All statements are presented in details below, but proofs are omitted since they are entirely analogous to the ones presented in Section 4. The definition of solution to 1.2 is given analogously to Definition 2.1, adding an obvious term related to the boundary $x=L$.

Definition 3.1. $A$ weak entropy solution to the IBVP 1.2 on the interval $[0, T]$ is a map $u \in$ $\mathbf{L}^{\infty}([0, T] \times[0, L] ; \mathbb{R})$, such that for any $k \in \mathbb{R}$ and for any test function $\varphi \in \mathbf{C}_{c}^{1}\left(\mathbb{R} \times \mathbb{R} ; \mathbb{R}_{+}\right)$ satisfies the following entropy inequalities

$$
\begin{aligned}
& \int_{0}^{T} \int_{0}^{L}\left\{\eta_{k}^{+}(u(t, x)) \partial_{t} \varphi(t, x)+\Phi_{k}^{+}(t, u(t, x)) \partial_{x} \varphi(t, x)\right\} \mathrm{d} x \mathrm{~d} t \\
& +\int_{0}^{L} \eta_{k}^{+}\left(u_{o}(x)\right) \varphi(0, x) \mathrm{d} x-\int_{0}^{L} \eta_{k}^{+}(u(T, x)) \varphi(T, x) \mathrm{d} x \\
& +\left\|\partial_{u} f\right\|_{\mathbf{L}^{\infty}([0, T] \times \mathcal{U} ; \mathbb{R})}\left(\int_{0}^{T} \eta_{k}^{+}\left(u_{b, 1}(t)\right) \varphi(t, 0) \mathrm{d} t+\int_{0}^{T} \eta_{k}^{+}\left(u_{b, 2}(t)\right) \varphi(t, L) \mathrm{d} t\right) \geq 0,
\end{aligned}
$$

and

$$
\begin{aligned}
& \int_{0}^{T} \int_{0}^{L}\left\{\eta_{k}^{-}(u(t, x)) \partial_{t} \varphi(t, x)+\Phi_{k}^{-}(t, u(t, x)) \partial_{x} \varphi(t, x)\right\} \mathrm{d} x \mathrm{~d} t \\
& +\int_{0}^{L} \eta_{k}^{-}\left(u_{o}(x)\right) \varphi(0, x) \mathrm{d} x-\int_{0}^{L} \eta_{k}^{-}(u(T, x)) \varphi(T, x) \mathrm{d} x \\
& +\left\|\partial_{u} f\right\|_{\mathbf{L}^{\infty}([0, T] \times \mathcal{U} ; \mathbb{R})}\left(\int_{0}^{T} \eta_{k}^{-}\left(u_{b, 1}(t)\right) \varphi(t, 0) \mathrm{d} t+\int_{0}^{T} \eta_{k}^{-}\left(u_{b, 2}(t)\right) \varphi(t, L) \mathrm{d} t\right) \geq 0,
\end{aligned}
$$

where $\mathcal{U}=\mathcal{U}\left(u_{o}, u_{b, 1_{\mid[0, T]}}, u_{b, 2}[0, T]\right.$ as in 2.2 .

Throughout, we denote $\boldsymbol{u}_{\boldsymbol{b}}=\left(u_{b, 1}, u_{b, 2}\right)$ and $\boldsymbol{w}_{\boldsymbol{b}}=\left(w_{b, 1}, w_{b, 2}\right)$. 
Proposition 3.2. Let $f \in \mathbf{C}^{1}([0, T] \times \mathbb{R} ; \mathbb{R})$ be such that $\left\{u \mapsto \partial_{t} f(t . u)\right\} \in \mathbf{W}_{\text {loc }}^{1, \infty}(\mathbb{R} ; \mathbb{R})$ for all $t \in[0, T], u_{o}, w_{o} \in\left(\mathbf{L}^{1} \cap \mathbf{L}^{\infty}\right)([0, L] ; \mathbb{R})$ and $\boldsymbol{u}_{\boldsymbol{b}}, \boldsymbol{w}_{\boldsymbol{b}} \in\left(\mathbf{L}^{1} \cap \mathbf{L}^{\infty}\right)\left([0, T] ; \mathbb{R}^{2}\right)$. Let $u, w \in \mathbf{L}^{\infty}([0, L] \times[0, T] ; \mathbb{R})$ solve the IBVP $(1.2)$, with data $\left(u_{o}, \boldsymbol{u}_{\boldsymbol{b}}\right)$ and $\left(w_{o}, \boldsymbol{w}_{\boldsymbol{b}}\right)$ respectively, in the sense of Definition 3.1, with $u$ and $w$ that both admit a trace for $x \rightarrow 0+$ and for $x \rightarrow L-$ for a.e. $t \in[0, T]$. Then, for all $t \in[0, T]$,

$\|u(t)-w(t)\|_{\mathbf{L}^{1}([0, L] ; \mathbb{R})} \leq\left\|u_{o}-w_{o}\right\|_{\mathbf{L}^{1}\left(\mathbb{R}_{+}[0, L] ; \mathbb{R}\right)}+\left\|\partial_{u} f\right\|_{\mathbf{L}^{\infty}([0, t] \times \mathcal{U} ; \mathbb{R})} \sum_{i=1}^{2}\left\|u_{b, i}-w_{b, i}\right\|_{\mathbf{L}^{1}([0, t] ; \mathbb{R})}$,

where $\mathcal{U}=\mathcal{U}\left(\boldsymbol{u}_{\boldsymbol{b} \mid[0, t]}, \boldsymbol{w}_{\boldsymbol{b} \mid[0, t]}\right)$ is as in 2.2 .

Along the lines of the preceding sections, we present first the results for a time independent flux and then those related to the non autonomous case. We provide all those details where the present results differ from those of sections 2.1 and 2.2 .

\subsection{The Autonomous Case on the Segment}

Consider the following autonomous IBVP, which is a particular case of 1.2 ):

$$
\left\{\begin{array}{lc}
\partial_{t} u+\partial_{x} f(u)=0 & (t, x) \in[0, T] \times[0, L] \\
u(0, x)=u_{o}(x) & x \in[0, L] \\
(u(t, 0), u(t, L))=\boldsymbol{u}_{\boldsymbol{b}}(t) & t \in[0, T]
\end{array}\right.
$$

Solutions to (3.1) are understood in the sense of Definition 3.1. Observe that Proposition 3.3 applies to (3.1), under the hypothesis $f \in \mathbf{C}^{1}(\mathbb{R} ; \mathbb{R})$.

The next Proposition ensures the existence of solutions to (3.1), as well as some of their properties, and it is the analogue to Proposition 2.3, with minor modifications in the estimates.

Proposition 3.3. Let $f \in \mathbf{W}_{\text {loc }}^{1, \infty}(\mathbb{R} ; \mathbb{R}), u_{o} \in\left(\mathbf{L}^{1} \cap \mathbf{B V}\right)([0, L] ; \mathbb{R}), \boldsymbol{u}_{\boldsymbol{b}} \in\left(\mathbf{L}^{1} \cap \mathbf{B V}\right)\left([0, T] ; \mathbb{R}^{2}\right)$. Then, problem (3.1) admits a solution $u$ in the sense of Definition 3.1, with the properties:

1. If $u_{o}$ and $\boldsymbol{u}_{\boldsymbol{b}}$ are piecewise constant, then for $t$ small, the map $t \rightarrow u(t)$ coincides with the gluing of Lax solutions to Riemann problems at the points of jumps of $u_{o}$, at $x=0$ and at $x=L$.

2. Range of $u$ : with the notation in $(2.2), u(t, x) \in \mathcal{U}\left(u_{o}, \boldsymbol{u}_{\boldsymbol{b} \mid[0, t]}\right)$ for a.e. $(t, x) \in[0, T] \times$ $[0, L]$. Hence, for all $t \in[0, T]$,

$$
\|u(t)\|_{\mathbf{L}^{\infty}([0, L] ; \mathbb{R})} \leq \max \left\{\left\|u_{o}\right\|_{\mathbf{L}^{\infty}([0, L] ; \mathbb{R})},\left\|u_{b, 1}\right\|_{\mathbf{L}^{\infty}([0, t] ; \mathbb{R})},\left\|u_{b, 2}\right\|_{\mathbf{L}^{\infty}([0, t] ; \mathbb{R})}\right\} .
$$

3. $u$ is Lipschitz continuous in time: for all $t_{1}, t_{2} \in[0, T]$,

$$
\begin{gathered}
\left\|u\left(t_{1}\right)-u\left(t_{2}\right)\right\|_{\mathbf{L}^{1}([0, L] ; \mathbb{R})} \leq C\left(u_{o}, \boldsymbol{u}_{\boldsymbol{b}}\right)\left\|f^{\prime}\right\|_{\mathbf{L}^{\infty}(\mathcal{U} ; \mathbb{R})}\left|t_{2}-t_{1}\right|, \\
C\left(u_{o}, \boldsymbol{u}_{\boldsymbol{b}}\right)=\operatorname{TV}\left(u_{o}\right)+\operatorname{TV}\left(\boldsymbol{u}_{\boldsymbol{b}} ;\left[0, t_{1} \vee t_{2}\right]\right)+\left|u_{b, 1}(0+)-u_{o}(0+)\right|+\left|u_{b, 2}(0+)-u_{o}(L-)\right| \\
\text { where } \left.\mathcal{U}=\mathcal{U}\left(u_{o}, \boldsymbol{u}_{\boldsymbol{b} \mid\left[0, t_{1} \vee t_{2}\right]}\right) \text {, with the notation } 2.2\right] .
\end{gathered}
$$


4. Total variation estimate: for all $t \in[0, T]$

$$
\operatorname{TV}(u(t)) \leq \operatorname{TV}\left(u_{o}\right)+\operatorname{TV}\left(\boldsymbol{u}_{\boldsymbol{b}} ;[0, t]\right)+\left|u_{b, 1}(0+)-u_{o}(0+)\right|+\left|u_{b, 2}(0+)-u_{o}(L-)\right| .
$$

We conclude this Section stating the stability of the solutions to (3.1) with respect to the flux, similarly to Theorem 2.4 .

Theorem 3.4. Let $f, g \in \mathbf{C}^{1}(\mathbb{R} ; \mathbb{R}), u_{o} \in\left(\mathbf{L}^{1} \cap \mathbf{B V}\right)([0, L] ; \mathbb{R})$ and $\boldsymbol{u}_{\boldsymbol{b}} \in\left(\mathbf{L}^{1} \cap \mathbf{B V}\right)\left([0, T] ; \mathbb{R}^{2}\right)$. Call $u$ and $v$ the solutions to the IBVP (3.1), with flux $f$ and $g$ respectively, constructed in Proposition 3.3. Then, with $\mathcal{U}=\mathcal{U}\left(u_{o}, \boldsymbol{u}_{\boldsymbol{b} \mid[0, t]}\right)$ as in $[2.2$, for all $t \in[0, T]$,

$$
\begin{aligned}
& \|u(t)-v(t)\|_{\mathbf{L}^{1}([0, L] ; \mathbb{R})} \\
\leq & \max \left\{1,\left\|g^{\prime}\right\|_{\mathbf{L}^{\infty}(\mathcal{U} ; \mathbb{R})}\right\}\|D(f-g)\|_{\mathbf{L}^{\infty}(\mathcal{U} ; \mathbb{R})} \\
& \times\left(\operatorname{TV}\left(u_{o}\right)+\operatorname{TV}\left(\boldsymbol{u}_{\boldsymbol{b}} ;[0, t]\right)+\left|u_{b, 1}(0+)-u_{o}(0+)\right|+\left|u_{b, 2}(0+)-u_{o}(L-)\right|\right) t .
\end{aligned}
$$

\subsection{The Non Autonomous Case on the Segment}

We now extend the results obtained in Section 3.1 to problem 1.2 .

Proposition 3.5. Let $f$ satisfy $(2.10)$. Fix $u_{o} \in\left(\mathbf{L}^{1} \cap \mathbf{B V}\right)([0, L] ; \mathbb{R})$ and $\boldsymbol{u}_{\boldsymbol{b}} \in\left(\mathbf{L}^{1} \cap\right.$ BV $)\left([0, T] ; \mathbb{R}^{2}\right)$. Then, problem 1.2 admits a solution $u$ in the sense of Definition 3.1, with:

1. Range of $u$ : with the notation in $(2.2), u(t, x) \in \mathcal{U}\left(u_{o}, \boldsymbol{u}_{\boldsymbol{b} \mid[0, t]}\right)$ for a.e. $(t, x) \in[0, T] \times$ $[0, L]$. Hence, for all $t \in[0, T]$,

$$
\|u(t)\|_{\mathbf{L}^{\infty}([0, L] ; \mathbb{R})} \leq \max \left\{\left\|u_{o}\right\|_{\mathbf{L}^{\infty}([0, L] ; \mathbb{R})},\left\|u_{b, 1}\right\|_{\mathbf{L}^{\infty}([0, t] ; \mathbb{R})},\left\|u_{b, 2}\right\|_{\mathbf{L}^{\infty}([0, t] ; \mathbb{R})}\right\} .
$$

2. $u$ is Lipschitz continuous in time: for all $t_{1}, t_{2} \in[0, T]$,

$$
\begin{gathered}
\left\|u\left(t_{1}\right)-u\left(t_{2}\right)\right\|_{\mathbf{L}^{1}([0, L] ; \mathbb{R})} \leq C\left(u_{o}, \boldsymbol{u}_{\boldsymbol{b}}\right)\left\|\partial_{u} f\right\|_{\mathbf{L}^{\infty}\left(\left[0, t_{1} \vee t_{2}\right] \times \mathcal{U} ; \mathbb{R}\right)}\left|t_{2}-t_{1}\right|, \\
C\left(u_{o}, \boldsymbol{u}_{\boldsymbol{b}}\right)=\operatorname{TV}\left(u_{o}\right)+\operatorname{TV}\left(\boldsymbol{u}_{\boldsymbol{b}} ;\left[0, t_{1} \vee t_{2}\right]\right)+\left|u_{b, 1}(0+)-u_{o}(0+)\right|+\left|u_{b, 2}(0+)-u_{o}(L-)\right| \\
\text { where } \left.\mathcal{U}=\mathcal{U}\left(u_{o}, \boldsymbol{u}_{\boldsymbol{b} \mid\left[0, t_{1} \vee t_{2}\right]}\right), \text { with the notation } 2.2\right)
\end{gathered}
$$

3. Total variation estimate: for all $t \in[0, T]$

$$
\operatorname{TV}(u(t)) \leq \operatorname{TV}\left(u_{o}\right)+\operatorname{TV}\left(\boldsymbol{u}_{\boldsymbol{b}} ;[0, t]\right)+\left|u_{b, 1}(0+)-u_{o}(0+)\right|+\left|u_{b, 2}(0+)-u_{o}(L-)\right| .
$$

We conclude this section with the analogue to Theorem 2.6, i.e. the stability of the solution to 1.2 with respect to the flux.

Theorem 3.6. Let $f, g$ satisfy $(2.10)$. Fix $u_{o} \in\left(\mathbf{L}^{1} \cap \mathbf{B V}\right)([0, L] ; \mathbb{R})$ and $\boldsymbol{u}_{\boldsymbol{b}} \in\left(\mathbf{L}^{1} \cap\right.$ $\mathbf{B V})\left([0, T] ; \mathbb{R}^{2}\right)$. Call $u, v$ the solutions to the IBVP 11.2 , with flux $f$ and $g$ respectively, constructed in Proposition 3.5. Then, with $\mathcal{U}=\mathcal{U}\left(u_{o}, \boldsymbol{u}_{\boldsymbol{b} \mid[0, t]}\right)$ as in $(2.2)$, for all $t \in[0, T]$,

$$
\begin{aligned}
& \|u(t)-v(t)\|_{\mathbf{L}^{1}([0, L] ; \mathbb{R})} \\
\leq & \max \left\{1,\left\|\partial_{u} g\right\|_{\mathbf{L}^{\infty}([0, t] \times \mathcal{U} ; \mathbb{R})}\right\}\left\|\partial_{u}(f-g)\right\|_{\mathbf{L}^{\infty}([0, t] \times \mathcal{U} ; \mathbb{R})} \\
& \times\left(\operatorname{TV}\left(u_{o}\right)+\operatorname{TV}\left(\boldsymbol{u}_{\boldsymbol{b}} ;[0, t]\right)+\left|u_{b, 1}(0+)-u_{o}(0+)\right|+\left|u_{b, 2}(0+)-u_{o}(L-)\right|\right) t .
\end{aligned}
$$




\section{Technical Proofs}

We distinguish between classical entropy-entropy flux pair and boundary entropy-entropy flux pair. In similar settings, the former notion, in the time independent case, is given in [9, Paragraph 7.4] or [15, Chapter 2, Definition 3.22], while for the latter we refer to [17, 18, see also [15, Chapter 2, Definition 7.1], [16, Definition 2] and [20, Definition 2]. We provide below the explicit definitions in the case of interest here, where $f=f(t, u)$.

Definition 4.1. The pair $(\eta, q) \in \mathbf{C}^{1}(\mathbb{R} ; \mathbb{R}) \times \mathbf{C}^{1}([0, T] \times \mathbb{R} ; \mathbb{R})$ is called a classical entropyentropy flux pair for the flux $f \in \mathbf{C}^{1}([0, T] \times \mathbb{R} ; \mathbb{R})$ if:

1. $\eta$ is convex;

2. for all $t \in[0, T]$ and all $u \in \mathbb{R}, \partial_{u} q(t, u)=\eta^{\prime}(u) \partial_{u} f(t, u)$.

Definition 4.2. The pair $(H, Q) \in \mathbf{C}^{1}\left(\mathbb{R}^{2} ; \mathbb{R}\right) \times \mathbf{C}^{1}\left([0, T] \times \mathbb{R}^{2} ; \mathbb{R}\right)$ is called a boundary entropy-entropy flux pair for the flux $f \in \mathbf{C}^{1}([0, T] \times \mathbb{R} ; \mathbb{R})$ if:

1. for all $w \in \mathbb{R}$, the function $u \mapsto H(u, w)$ is convex;

2. for all $t \in[0, T]$ and all $u, w \in \mathbb{R}, \partial_{u} Q(t, u, w)=\partial_{u} H(u, w) \partial_{u} f(t, u)$;

3. for all $t \in[0, T]$ and all $w \in \mathbb{R}, H(w, w)=0, Q(t, w, w)=0$ and $\partial_{u} H(w, w)=0$.

Consequences of Definition 2.1 are collected in the following lemmas, whose proofs directly follow from [20, Lemma 1 and Remark 3], see also [16, Lemma 3, Lemma 4 and Lemma 16].

Lemma 4.3. If $u \in \mathbf{L}^{\infty}\left([0, T] \times \mathbb{R}_{+} ; \mathbb{R}\right)$ is a weak entropy solution to (1.1) in the sense of Definition 2.1. then, for all classical entropy-entropy flux pairs $(\eta, q)$, for all $\varphi \in \mathbf{C}_{c}^{1}(\mathbb{R} \times$ $\left.\mathbb{R}_{+} ; \mathbb{R}_{+}\right)$,

$$
\begin{aligned}
& \int_{0}^{T} \int_{\mathbb{R}_{+}}\left\{\eta(u(t, x)) \partial_{t} \varphi(t, x)+q(t, u(t, x)) \partial_{x} \varphi(t, x)\right\} \mathrm{d} x \mathrm{~d} t \\
& \quad+\int_{\mathbb{R}_{+}} \eta\left(u_{o}(x)\right) \varphi(0, x) \mathrm{d} x-\int_{\mathbb{R}_{+}} \eta(u(T, x)) \varphi(T, x) \mathrm{d} x \geq 0 .
\end{aligned}
$$

In particular, for all $\varphi \in \mathbf{C}_{c}^{1}\left(\mathbb{R} \times \stackrel{\circ}{\mathbb{R}}_{+} ; \mathbb{R}_{+}\right)$and for all $k \in \mathbb{R}$,

$$
\begin{aligned}
& \int_{0}^{T} \int_{\mathbb{R}_{+}}\left\{|u(t, x)-k| \partial_{t} \varphi(t, x)\right. \\
& \left.\quad+\operatorname{sgn}(u(t, x)-k)(f(t, u(t, x))-f(t, k)) \partial_{x} \varphi(t, x)\right\} \mathrm{d} x \mathrm{~d} t \\
& +\int_{\mathbb{R}_{+}}\left|u_{o}(x)-k\right| \varphi(0, x) \mathrm{d} x-\int_{\mathbb{R}_{+}}|u(T, x)-k| \varphi(T, x) \mathrm{d} x \geq 0 .
\end{aligned}
$$

Lemma 4.4. If $u \in \mathbf{L}^{\infty}\left([0, T] \times \mathbb{R}_{+} ; \mathbb{R}\right)$ is a weak entropy solution to (1.1) in the sense of Definition 2.1. then, for all boundary entropy-entropy flux pair $(H, Q)$ and for all $\beta \in \mathbf{L}^{1}(\mathbb{R} ; \mathbb{R})$ with $\beta \geq 0$ a.e.,

$$
\underset{s \rightarrow 0^{+}}{\operatorname{ess} \lim _{0}} \int_{0}^{T} Q\left(t, u(t, s), u_{b}(t)\right) \beta(t) \mathrm{d} t \leq 0 .
$$

Moreover, if $u$ admits a trace $u(t, 0+)$ at $x=0$ for a.e. $t \in[0, T], 4.3$ is equivalent to

$$
\int_{0}^{T} Q\left(t, u(t, 0+), u_{b}(t)\right) \beta(t) \mathrm{d} t \leq 0 .
$$


We now extend part of [15, Chapter 2, Lemma 7.24] to the time dependent case.

Lemma 4.5. Let $u_{b} \in \mathbf{L}^{\infty}([0, T] ; \mathbb{R})$ and let $u \in \mathbf{L}^{\infty}([0, T] \times \mathbb{R} ; \mathbb{R})$ admit a trace $u(t, 0+)$ at $x=0$ for a.e. $t \in[0, T]$. If (4.4) holds, then for a.e. $t \in[0, T]$ and for all $k \in \mathcal{I}\left(u(t, 0+), u_{b}(t)\right)$ as in 2.1,

$$
\operatorname{sgn}\left(u(t, 0+)-u_{b}(t)\right)(f(t, u(t, 0+))-f(t, k)) \leq 0 .
$$

Proof. For all $k \in \mathbb{R}$ and for $n \in \mathbb{N} \backslash\{0\}$, define the maps

$$
\begin{aligned}
& \Delta^{k}(u, w)=\min _{z \in \mathcal{I}(w, k)}|u-z| \\
& \mathcal{F}^{k}(t, u, w)= \begin{cases}f(t, w)-f(t, u) & \text { for } u \leq w \leq k \\
0 & \text { for } w \leq u \leq k \\
f(t, u)-f(t, k) & \text { for } w \leq k \leq u \\
f(t, k)-f(t, u) & \text { for } u \leq k \leq w \\
0 & \text { for } k \leq u \leq w \\
f(t, u)-f(t, w) & \text { for } k \leq w \leq u\end{cases} \\
& H_{n}^{k}(u, w)= \\
& Q_{n}^{k}(t, u, w)= \int_{w}^{u} \partial_{u} H_{n}^{k}(z, w) \partial_{u} f(t, z) \mathrm{d} z .
\end{aligned}
$$

Clearly, for all $k \in \mathbb{R}$, the sequence of boundary entropy-entropy flux pairs $\left(H_{n}^{k}, Q_{n}^{k}\right)$ converges uniformly to $\left(\Delta^{k}, \mathcal{F}^{k}\right)$ as $n \rightarrow+\infty$. Applying (4.4) with $Q$ replaced by $Q_{n}^{k}$, in the limit $n \rightarrow+\infty$ yields that for all $k \in \mathbb{R}$ and for all $\beta \in \mathbf{L}^{1}(\mathbb{R} ; \mathbb{R})$ with $\beta \geq 0$ a.e.,

$$
\begin{aligned}
\int_{0}^{T} \mathcal{F}^{k}\left(t, u(t, 0+), u_{b}(t)\right) \beta(t) \mathrm{d} t & \leq 0 \\
\mathcal{F}^{k}\left(t, u(t, 0+), u_{b}(t)\right) & \leq 0 \quad \text { for a.e. } t \in[0, T] .
\end{aligned}
$$

Choose now $k \in \mathcal{I}\left(u(t, 0+), u_{b}(t)\right)$ so that, by (4.6), the bound (4.7) ensures 4.5).

Proof of Proposition 2.2. This proof closely follows that of [8, Theorem 4.3], but using the doubling of variables method as in [16, Lemma 17], which is consistent with the present Definition 2.1. Key points are the choice of an appropriate test function and the use of Lemma 4.4 and Lemma 4.5 .

Note that here there is no source term, the flux $f$ does not depend on the space variable and we are dealing with $\mathbb{R}_{+}$instead of a bounded domain $\Omega \subseteq \mathbb{R}^{n}$. A careful checking of the proof in [8] shows that the present assumptions on $f$ are sufficient.

\subsection{Proofs related to the Autonomous IBVP on the Half-Line}

Proof of Proposition 2.3. For $\varepsilon>0$, introduce the set $\mathbf{P C}\left(\mathbb{R}_{+} ; \varepsilon \mathbb{Z}\right)$ of maps $u$ of the form $u=\sum_{\alpha=1}^{N} u_{\alpha} \chi_{I_{\alpha}}$, where $N \in \mathbb{N}, u_{\alpha} \in \varepsilon \mathbb{Z}$ and $I_{\alpha}$ is a real interval for all $\alpha=1, \ldots, N$. $\operatorname{PLC}(\mathbb{R} ; \mathbb{R})$ is the set of real valued piecewise linear and continuous functions defined on $\mathbb{R}$. 
A.1) Construction of $\varepsilon$-approximate solutions. Following [ $[6$, Chapter 6], for any positive $\varepsilon$ introduce the following approximations:

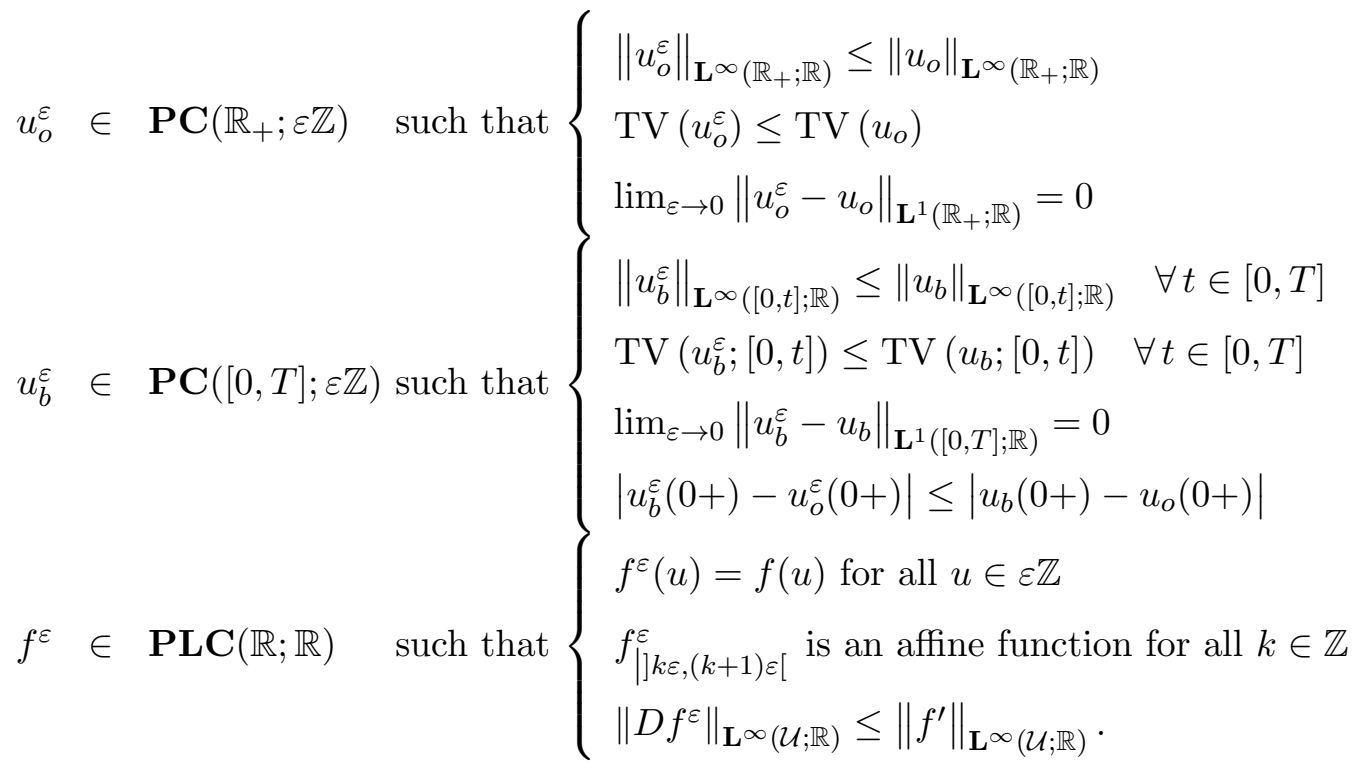

We approximate the solution to the original IBVP 2.8 with exact solutions $u_{\varepsilon}$ to the $\varepsilon^{-}$ approximate IBVPs

$$
\left\{\begin{array}{lc}
\partial_{t} u^{\varepsilon}+\partial_{x} f^{\varepsilon}\left(u^{\varepsilon}\right)=0 & (t, x) \in[0, T] \times \mathbb{R}_{+} \\
u^{\varepsilon}(0, x)=u_{o}^{\varepsilon}(x) & x \in \mathbb{R}_{+} \\
u^{\varepsilon}(t, 0)=u_{b}^{\varepsilon}(t) & t \in[0, T] .
\end{array}\right.
$$

At the initial time $t=0$, solving $(4.9)$ for $x>0$ amounts to glue the solutions to the Riemann problems at the points of jump in $u_{o}^{\varepsilon}$, see $[6, \S 6.1]$. A local solution at $(0,0)$ is obtained by restricting the solution to the Riemann problem for $f^{\varepsilon}$ with left and right state $u_{b}^{\varepsilon}(0)$ and $u_{o}^{\varepsilon}(0)$ respectively, see [1, Example C]. Recall from [6, Chapter 6] that, with the above choice of $f^{\varepsilon}$, the solutions to Riemann problems with data in $\varepsilon \mathbb{Z}$ still take values in the set $\varepsilon \mathbb{Z}$.

We thus have a piecewise constant solution $u^{\varepsilon}$ to 4.9 defined for $t>0$ sufficiently small. This solution can be prolonged up to the first time of interaction $t_{1}>0$ at which one of the following events takes place:

(i) two or more lines of discontinuity hit each other;

(ii) one wave hits the boundary $x=0$;

(iii) the value of the boundary condition $u_{b}^{\varepsilon}$ changes.

In case (i), it is possible to extend the solution beyond $t_{1}$ by solving the new Riemann problems generated by the interactions, as in [6, $\S 6.1]$. In cases (ii) and (iii), the extension beyond $t_{1}$ is achieved by restricting to $\mathbb{R}_{+}$the solution to the Riemann problem with left state $u_{b}^{\varepsilon}\left(t_{1}+\right)$ and right state $u^{\varepsilon}\left(t_{1}, 0+\right)$. The solution is then prolonged up to the next time of interaction $t_{2}>t_{1}$, and so on.

Note that, by construction, waves in $u^{\varepsilon}$ satisfy both Rankine-Hugoniot condition [9, Formula (4.3.5)] and Oleinik entropy condition [9, Formula (8.4.3)], in the sense that, whenever 
two states $u^{\ell}$ and $u^{r}$ in $u^{\varepsilon}$ are separated by a wave propagating with speed $\lambda$, we have

$$
\lambda=\frac{f^{\varepsilon}\left(u^{\ell}\right)-f^{\varepsilon}\left(u^{r}\right)}{u^{\ell}-u^{r}} \text { and } \frac{f^{\varepsilon}\left(u^{r}\right)-f^{\varepsilon}(k)}{u^{r}-k} \leq \lambda \leq \frac{f^{\varepsilon}(k)-f^{\varepsilon}\left(u^{\ell}\right)}{k-u^{\ell}} \quad \forall k \in \mathcal{I}\left(u^{\ell}, u^{r}\right) .
$$

Moreover, the above conditions 4.10 impose that, whenever $u^{\varepsilon}(t, 0+)=u^{r}$, we have that

$$
\text { if } u^{r} \neq u_{b}^{\varepsilon}(t) \quad \text { then } \quad \frac{f^{\varepsilon}\left(u^{r}\right)-f^{\varepsilon}(k)}{u^{r}-k} \leq 0 \quad \forall k \in \mathcal{I}\left(u_{b}^{\varepsilon}(t), u^{r}\right) .
$$

A.2) Wave Front Tracking Solutions are Weak Entropy Solutions. By standard arguments, it is sufficient to verify (2.6) and (2.7) in the following two cases:

1. The support of the (positive) test function $\varphi$ is contained in $\left.\left[t_{1}, t_{2}\right] \times\left[x_{1}, x_{2}\right] \subset\right] 0, T\left[\times \mathbb{R}_{+}\right.$ and here the wave front tracking solution $u^{\varepsilon}$ attains only the two values $u^{\ell}$ and $u^{r}$, separated by a wave with speed $\lambda=\frac{x_{2}-x_{1}}{t_{2}-t_{1}}$.

2. The support of the (positive) test function $\varphi$ is contained in $\left[t_{1}, t_{2}\right] \times\left[x_{1}, x_{2}\right]$ with $x_{1}<$ $0<x_{2}$, the boundary data satisfies $u_{b}^{\varepsilon}(t)=u^{\ell}$ for $t \in\left[t_{1}, t_{2}\right]$ and $u^{\varepsilon}(t, x)=u^{r}$ for $(t, x) \in$ $\left.\left.\left[t_{1}, t_{2}\right] \times\right] 0, x_{2}\right]$.

The other cases, that of a single wave with negative speed, of interacting waves, of waves interacting with the boundary and of the boundary datum changing value, can be recovered through manipulations of the test functions and immediate modifications of $\mathbf{1}$. and $\mathbf{2}$.

1. Assume $k<u^{\ell}<u^{r}$. Then, direct computations show that 2.6 is equivalent to

$$
\left[\lambda\left(u^{r}-u^{\ell}\right)-\left(f^{\varepsilon}\left(u^{r}\right)-f^{\varepsilon}\left(u^{\ell}\right)\right)\right] \int_{t_{1}}^{t_{2}} \varphi\left(t, x_{1}+\lambda\left(t-t_{1}\right)\right) \mathrm{d} t \geq 0,
$$

which holds since the left hand side vanishes by the Rankine-Hugoniot condition 4.10). It is immediate to check that the left hand side in (2.7) vanishes.

If $u^{\ell}<k<u^{r}$, then 2.6) is equivalent to

$$
\left(u^{r}-k\right)\left(\lambda-\frac{f^{\varepsilon}\left(u^{\ell}\right)-f^{\varepsilon}\left(u^{r}\right)}{u^{\ell}-u^{r}}\right) \int_{t_{1}}^{t_{2}} \varphi\left(t, x_{1}+\lambda\left(t-t_{1}\right)\right) \mathrm{d} t \geq 0,
$$

which holds by Oleinik entropy condition 4.10). On the other hand, 2.7 is equivalent to

$$
\left(k-u^{\ell}\right)\left(\frac{f^{\varepsilon}(k)-f^{\varepsilon}\left(u^{\ell}\right)}{k-u^{\ell}}-\lambda\right) \int_{t_{1}}^{t_{2}} \varphi\left(t, x_{1}+\lambda\left(t-t_{1}\right)\right) \mathrm{d} t \geq 0,
$$

which again holds by Oleinik entropy condition (4.10).

If $u^{\ell}<u^{r}<k$, then 2.7 is equivalent to (4.12), while the left hand side in 2.6) vanishes.

The cases $k<u^{r}<u^{\ell}, u^{r}<k<u^{\ell}$ and $u^{r}<u^{\ell}<k$ are entirely analogous.

2. Assume $k<u^{\ell}<u^{r}$. Then, direct computations show that 2.6 is equivalent to

$$
\left[-\left(f^{\varepsilon}\left(u^{r}\right)-f^{\varepsilon}(k)\right)+\left\|D f^{\varepsilon}\right\|_{\mathbf{L}^{\infty}(\mathcal{U} ; \mathbb{R})}\left(u^{\ell}-k\right)\right] \int_{t_{1}}^{t_{2}} \varphi(t, 0) \mathrm{d} t \geq 0 .
$$

Note that, by the Lipschitz continuity of $f^{\varepsilon}$, we have

$$
-\left(f^{\varepsilon}\left(u^{r}\right)-f^{\varepsilon}(k)\right)+\left\|D f^{\varepsilon}\right\|_{\mathbf{L}^{\infty}(\mathcal{U} ; \mathbb{R})}\left(u^{\ell}-k\right) \geq f^{\varepsilon}\left(u^{\ell}\right)-f^{\varepsilon}\left(u^{r}\right)
$$


and the latter term above is non negative by (4.11). Hence, the left hand side in (2.7) vanishes.

Assume $u^{\ell}<k<u^{r}$. Then, (2.6) is equivalent to

$$
\left(f^{\varepsilon}(k)-f^{\varepsilon}\left(u^{r}\right)\right) \int_{t_{1}}^{t_{2}} \varphi(t, 0) \mathrm{d} t \geq 0,
$$

which holds by Oleinik entropy condition (4.11). Hence, 2.7) reads

$$
\left\|D f^{\varepsilon}\right\|_{\mathbf{L}^{\infty}(\mathcal{U} ; \mathbb{R})}\left(k-u^{\ell}\right) \int_{t_{1}}^{t_{2}} \varphi(t, 0) \mathrm{d} t \geq 0
$$

and this inequality clearly holds.

Assume $u^{\ell}<u^{r}<k$. Then, the left hand side in 2.6) vanishes. Condition (2.7) becomes

$$
\left[\left(f^{\varepsilon}\left(u^{r}\right)-f^{\varepsilon}(k)\right)+\left\|D f^{\varepsilon}\right\|_{\mathbf{L}^{\infty}(\mathcal{U} ; \mathbb{R})}\left(k-u^{\ell}\right)\right] \int_{t_{1}}^{t_{2}} \varphi(t, 0) \mathrm{d} t \geq 0 .
$$

Note that, by the Lipschitz continuity of $f^{\varepsilon}$, we have

$$
\left(f^{\varepsilon}\left(u^{r}\right)-f^{\varepsilon}(k)\right)+\left\|D f^{\varepsilon}\right\|_{\mathbf{L}^{\infty}(\mathcal{U} ; \mathbb{R})}\left(k-u^{\ell}\right) \geq\left\|D f^{\varepsilon}\right\|_{\mathbf{L}^{\infty}(\mathcal{U} ; \mathbb{R})}\left(u^{r}-u^{\ell}\right)
$$

and the latter term above is non negative in the present case.

A.3) The map $t \rightarrow \operatorname{TV}\left(u^{\varepsilon}(t)\right)$ is uniformly bounded, as long as $u^{\varepsilon}$ is defined. Introduce for $t \in[0, T]$, the Glimm functional

$$
V^{\varepsilon}(t)=\operatorname{TV}\left(u^{\varepsilon}(t)\right)+\operatorname{TV}\left(u_{b}^{\varepsilon} ;[t, T]\right)+\left|u_{b}^{\varepsilon}(t+)-u^{\varepsilon}(t, 0+)\right| .
$$

Clearly, TV $\left(u^{\varepsilon}(t)\right) \leq V^{\varepsilon}(t)$. We claim that $t \rightarrow V^{\varepsilon}(t)$ is non increasing. Indeed, at an interaction time, the proof in [6, $\S 6.1]$ applies in case (i) in Step A.1, while minor modifications yield the proof in the other two cases (ii) and (iii). The inequality $V^{\varepsilon}(t) \leq V^{\varepsilon}(0)$ implies

$$
\operatorname{TV}\left(u^{\varepsilon}(t)\right) \leq \operatorname{TV}\left(u_{o}^{\varepsilon}\right)+\operatorname{TV}\left(u_{b}^{\varepsilon} ;[0, t]\right)+\left|u_{b}^{\varepsilon}(0+)-u_{o}^{\varepsilon}(0+)\right| .
$$

A.4) The total number of interactions is finite and $u^{\varepsilon}$ is defined for all $t \in[0, T]$. When $t$ is not a time of interaction, define the weighted number of discontinuities in $u^{\varepsilon}(t)$ as

$$
\begin{aligned}
\sharp(t)= & {\left[\text { number of discontinuities in } u^{\varepsilon}(t)\right] } \\
& +2 \frac{\left\|u_{b}\right\|_{\mathbf{L}^{\infty}\left(\mathbb{R}_{+} ; \mathbb{R}\right)}+\left\|u_{o}\right\|_{\mathbf{L}^{\infty}\left(\mathbb{R}_{+} ; \mathbb{R}\right)}}{\varepsilon}\left[\text { number of discontinuities in } \mathscr{T}_{t} u_{b}^{\varepsilon}\right] \\
& +\frac{1}{\varepsilon}\left|u_{b}^{\varepsilon}(t)-u^{\varepsilon}(t, 0)\right|,
\end{aligned}
$$

where we used the notation (2.3). If $t$ is an interaction time, set $\sharp(t)=\lim _{\tau \rightarrow t+} \sharp(\tau)$.

The procedure in $[6, \S 6.1]$ can be applied, ensuring that at those interaction times where $\sharp$ increases, $V^{\varepsilon}$ diminishes by at least $\varepsilon$.

A.5) Range of $u^{\varepsilon}$. At any interaction time $t_{*}$, the new values attained by $u^{\varepsilon}$ lie in the convex hull of the values attained by $u^{\varepsilon}$ before time $t_{*}$, proving that $u^{\varepsilon}(t, x) \subseteq \mathcal{U}\left(u_{o}, u_{b \mid[0, t]}\right)$ for a.e. $(t, x) \in[0, T] \times \mathbb{R}_{+}$, with the notation (2.2). It is then immediate to verify that at any time $t \in[0, T]$

$$
\left\|u^{\varepsilon}(t)\right\|_{\mathbf{L}^{\infty}\left(\mathbb{R}_{+} ; \mathbb{R}\right)} \leq \max \left\{\left\|u_{o}\right\|_{\mathbf{L}^{\infty}\left(\mathbb{R}_{+} ; \mathbb{R}\right)},\left\|u_{b}\right\|_{\mathbf{L}^{\infty}([0, t] ; \mathbb{R})}\right\} .
$$


A.6) $\mathbf{L}^{1}-$ Lipschitz continuity of $t \rightarrow u^{\varepsilon}(t)$. Assume that $t_{2}>t_{1}$. Observe first that $u^{\varepsilon}$ remains unaltered on the interval $\left[0, t_{2}\right]$ if $u_{b}^{\varepsilon}$ is substituted by $\tilde{u}_{b}^{\varepsilon}=u_{b}^{\varepsilon} \chi_{\left[0, t_{2}\right]}+u_{b}^{\varepsilon}\left(t_{2}+\right) \chi_{\left[t_{2}, T\right]^{\circ}}$. At any interaction time $t_{*}$, if $t_{1}<t_{*}<t_{2}$ and $t_{2}-t_{1}$ is sufficiently small, denoting $\mathcal{U}=$ $\mathcal{U}\left(u_{o}, u_{b \mid\left[0, t_{2}\right]}\right)$ as in $(2.2)$,

$$
\begin{aligned}
& \left\|u^{\varepsilon}\left(t_{2}\right)-u^{\varepsilon}\left(t_{1}\right)\right\|_{\mathbf{L}^{1}\left(\mathbb{R}_{+} ; \mathbb{R}\right)} \\
& \leq\left\|f^{\prime}\right\|_{\mathbf{L}^{\infty}(\mathcal{U} ; \mathbb{R})} V^{\varepsilon}\left(t_{*}\right)\left|t_{2}-t_{1}\right| \quad \text { [by [6, Formula (6.14)] and 4.14] ] } \\
& \leq\left\|f^{\prime}\right\|_{\mathbf{L}^{\infty}(\mathcal{U} ; \mathbb{R})} V^{\varepsilon}(0)\left|t_{2}-t_{1}\right| \quad \text { [since } t \rightarrow V^{\varepsilon}(t) \text { is non increasing] } \\
& =\left\|f^{\prime}\right\|_{\mathbf{L}^{\infty}(\mathcal{U} ; \mathbb{R})}\left(\operatorname{TV}\left(u_{o}^{\varepsilon}\right)+\operatorname{TV}\left(\tilde{u}_{b}^{\varepsilon}\right)+\left|\tilde{u}_{b}^{\varepsilon}(0+)-u_{o}^{\varepsilon}(0+)\right|\right)\left|t_{2}-t_{1}\right| \quad \text { [by 4.13] ] } \\
& =\left\|f^{\prime}\right\|_{\mathbf{L}^{\infty}(\mathcal{U} ; \mathbb{R})}\left(\operatorname{TV}\left(u_{o}^{\varepsilon}\right)+\operatorname{TV}\left(u_{b}^{\varepsilon} ;\left[0, t_{2}\right]\right)+\left|u_{b}^{\varepsilon}(0+)-u_{o}^{\varepsilon}(0+)\right|\right)\left|t_{2}-t_{1}\right| .
\end{aligned}
$$

A.7) Existence of a Solution. By Helly Theorem [6, Theorem 2.4], for any sequence $\varepsilon_{n}$ converging to 0 , the sequence $u^{\varepsilon_{n}}$ converges pointwise almost everywhere, up to a subsequence, to a map $u$. We now show that this limit function $u$ is a weak entropy solution to (2.8), in the sense of Definition 2.1. Any $u^{\varepsilon_{n}}$ is a weak entropy solution to $(4.9)$ by Step A.2; hence, $u^{\varepsilon_{n}}$ satisfies for any $k \in \mathbb{R}$ and for any test function $\varphi \in \mathbf{C}_{c}^{1}(\mathbb{R} \times \mathbb{R} ; \mathbb{R})$ the two entropy inequalities

$$
\begin{aligned}
0 \leq & \int_{0}^{T} \int_{\mathbb{R}_{+}}\left\{\eta_{k}^{ \pm}\left(u^{\varepsilon_{n}}(t, x)\right) \partial_{t} \varphi(t, x)+\Phi_{k, n}^{ \pm}\left(u^{\varepsilon_{n}}(t, x)\right) \partial_{x} \varphi(t, x)\right\} \mathrm{d} x \mathrm{~d} t \\
& +\int_{\mathbb{R}_{+}} \eta_{k}^{ \pm}\left(u_{o}^{\varepsilon_{n}}(x)\right) \varphi(0, x) \mathrm{d} x-\int_{\mathbb{R}_{+}} \eta_{k}^{ \pm}\left(u^{\varepsilon_{n}}(T, x)\right) \varphi(T, x) \mathrm{d} x \\
& +\left\|D f^{\varepsilon_{n}}\right\|_{\mathbf{L}^{\infty}(\mathcal{U} ; \mathbb{R})} \int_{0}^{T} \eta_{k}^{ \pm}\left(u_{b}^{\varepsilon_{n}}(t)\right) \varphi(t, 0) \mathrm{d} t,
\end{aligned}
$$

where $\eta_{k}^{ \pm}$and $\Phi_{k, n}^{ \pm}$are defined as in (2.5), using the autonomous flux function $f^{\varepsilon_{n}}$.

Consider each term separately. Since $\eta_{k}^{ \pm}$are Lipschitz continuous function with Lipschitz constant 1, we can estimate the first term in 4.17) as follows:

$$
\begin{aligned}
& \int_{0}^{T} \int_{\mathbb{R}_{+}} \eta_{k}^{ \pm}\left(u^{\varepsilon_{n}}(t, x)\right) \partial_{t} \varphi(t, x) \mathrm{d} x \mathrm{~d} t \\
= & \int_{0}^{T} \int_{\mathbb{R}_{+}} \eta_{k}^{ \pm}(u(t, x)) \partial_{t} \varphi(t, x) \mathrm{d} x \mathrm{~d} t+\int_{0}^{T} \int_{\mathbb{R}_{+}}\left(\eta_{k}^{ \pm}\left(u^{\varepsilon_{n}}(t, x)\right)-\eta_{k}^{ \pm}(u(t, x))\right) \partial_{t} \varphi(t, x) \mathrm{d} x \mathrm{~d} t \\
\leq & \int_{0}^{T} \int_{\mathbb{R}_{+}} \eta_{k}^{ \pm}(u(t, x)) \partial_{t} \varphi(t, x) \mathrm{d} x \mathrm{~d} t+\int_{0}^{T} \int_{\mathbb{R}_{+}}\left|u^{\varepsilon_{n}}(t, x)-u(t, x)\right| \partial_{t} \varphi(t, x) \mathrm{d} x \mathrm{~d} t
\end{aligned}
$$

and the second addend in 4.20 goes to 0 as $\varepsilon_{n}$ goes to 0.

Concerning the second term in (4.17), proceed as follows:

$$
\begin{aligned}
& \int_{0}^{T} \int_{\mathbb{R}_{+}} \Phi_{k, n}^{ \pm}\left(u^{\varepsilon_{n}}(t, x)\right) \partial_{x} \varphi(t, x) \mathrm{d} x \mathrm{~d} t \\
\leq & \int_{0}^{T} \int_{\mathbb{R}_{+}} \Phi_{k}^{ \pm}(u(t, x)) \partial_{x} \varphi(t, x) \mathrm{d} x \mathrm{~d} t
\end{aligned}
$$




$$
\begin{aligned}
& \quad+\int_{0}^{T} \int_{\mathbb{R}_{+}}\left(\Phi_{k, n}^{ \pm}(u(t, x))-\Phi_{k}^{ \pm}(u(t, x))\right) \partial_{x} \varphi(t, x) \mathrm{d} x \mathrm{~d} t \\
& +\int_{0}^{T} \int_{\mathbb{R}_{+}}\left(\Phi_{k, n}^{ \pm}\left(u^{\varepsilon_{n}}(t, x)\right)-\Phi_{k, n}^{ \pm}(u(t, x))\right) \partial_{x} \varphi(t, x) \mathrm{d} x \mathrm{~d} t \\
& \leq \int_{0}^{T} \int_{\mathbb{R}_{+}} \Phi_{k}^{ \pm}(u(t, x)) \partial_{x} \varphi(t, x) \mathrm{d} x \mathrm{~d} t \\
& +\int_{0}^{T} \int_{\mathbb{R}_{+}} \operatorname{sgn}^{ \pm}(u-k)\left(f^{\varepsilon_{n}}(u(t, x))-f^{\varepsilon_{n}}(k)-f(u(t, x))+f(k)\right) \partial_{x} \varphi(t, x) \mathrm{d} x \mathrm{~d} t \\
& +\left\|D f^{\varepsilon_{n}}\right\|_{\mathbf{L}^{\infty}(\mathcal{U} ; \mathbb{R})} \int_{0}^{T} \int_{\mathbb{R}_{+}}\left|u^{\varepsilon_{n}}(t, x)-u(t, x)\right| \partial_{x} \varphi(t, x) \mathrm{d} x \mathrm{~d} t
\end{aligned}
$$

and, as $\varepsilon_{n}$ tends to $0,4.22$ goes to 0 since $f^{\varepsilon_{n}}$ converges uniformly to $f$, while 4.23 vanishes in the limit due to the convergence of $u^{\varepsilon_{n}}$ to $u$.

The two terms in (4.18) are treated in the same way:

$$
\begin{aligned}
& \int_{\mathbb{R}_{+}} \eta_{k}^{ \pm}\left(u_{o}^{\varepsilon_{n}}(x)\right) \varphi(0, x) \mathrm{d} x-\int_{\mathbb{R}_{+}} \eta_{k}^{ \pm}\left(u^{\varepsilon_{n}}(T, x)\right) \varphi(T, x) \mathrm{d} x \\
= & \int_{\mathbb{R}_{+}} \eta_{k}^{ \pm}\left(u_{o}(x)\right) \varphi(0, x) \mathrm{d} x-\int_{\mathbb{R}_{+}} \eta_{k}^{ \pm}(u(T, x)) \varphi(T, x) \mathrm{d} x \\
& +\int_{\mathbb{R}_{+}}\left(\eta_{k}^{ \pm}\left(u_{o}^{\varepsilon_{n}}(x)\right)-\eta_{k}^{ \pm}\left(u_{o}(x)\right)\right) \varphi(0, x) \mathrm{d} x \\
& -\int_{\mathbb{R}_{+}}\left(\eta_{k}^{ \pm}\left(u^{\varepsilon_{n}}(T, x)\right)-\eta_{k}^{ \pm}(u(T, x))\right) \varphi(T, x) \mathrm{d} x
\end{aligned}
$$

and, since $\eta_{k}^{ \pm}$are Lipschitz continuous with constant 1, 4.25) and (4.26) vanish as $\varepsilon_{n}$ goes to 0 , due to the assumptions (4.8) on the initial datum and to the fact that $u^{\varepsilon_{n}}$ converges to $u$.

Pass now to 4.19). Thanks to $\left\|D f^{\varepsilon_{n}}\right\|_{\mathbf{L}^{\infty}(\mathcal{U} ; \mathbb{R})} \leq\left\|f^{\prime}\right\|_{\mathbf{L}^{\infty}(\mathcal{U} ; \mathbb{R})}$, see 44.8), we obtain

$$
\begin{aligned}
& \left\|D f^{\varepsilon_{n}}\right\|_{\mathbf{L}^{\infty}(\mathcal{U} ; \mathbb{R})} \int_{0}^{T} \eta_{k}^{ \pm}\left(u_{b}^{\varepsilon_{n}}(t)\right) \varphi(t, 0) \mathrm{d} t \\
\leq & \left\|f^{\prime}\right\|_{\mathbf{L}^{\infty}(\mathcal{U} ; \mathbb{R})} \int_{0}^{T} \eta_{k}^{ \pm}\left(u_{b}(t)\right) \varphi(t, 0) \mathrm{d} t \\
& +\left\|f^{\prime}\right\|_{\mathbf{L}^{\infty}(\mathcal{U} ; \mathbb{R})} \int_{0}^{T}\left(\eta_{k}^{ \pm}\left(u_{b}^{\varepsilon_{n}}(t)\right)-\eta_{k}^{ \pm}\left(u_{b}(t)\right)\right) \varphi(t, 0) \mathrm{d} t
\end{aligned}
$$

and (4.28) vanishes as $\varepsilon_{n}$ goes to 0 , thanks to the Lipschitz continuity of $\eta_{k}^{ \pm}$and the assumptions (4.8) on the boundary datum.

Collecting together the results above, in the limit $\varepsilon_{n} \rightarrow 0$, we obtain that $u$ is a weak entropy solution to 2.8 .

A.8) Conclusion. Point 1. holds by construction. For a.e. $(t, x) \in[0, T] \times \mathbb{R}_{+}, u^{\varepsilon}(t, x) \subseteq$ $\mathcal{U}\left(u_{o}, u_{b \mid[0, t]}\right)$ and 4.15 imply Point 2. Formula 4.16) and the assumptions 4.8) on the $\varepsilon$-approximation ensure Point 3. finally, Point 4. follows from the inequalities

$$
\operatorname{TV}(u(t))+\operatorname{TV}\left(u_{b} ;[t, T]\right)
$$




$$
\begin{aligned}
& \leq \lim _{\varepsilon \rightarrow 0}\left(\operatorname{TV}\left(u^{\varepsilon}(t)\right)+\operatorname{TV}\left(u_{b}^{\varepsilon}(t) ;[t, T]\right)\right) \quad \text { [lower semicontinuity of the TV] } \\
& \leq \lim _{\varepsilon \rightarrow 0} V^{\varepsilon}(t) \quad[\text { see 4.13)] } \\
& \leq \lim _{\varepsilon \rightarrow 0} V^{\varepsilon}(0) \\
& \leq \operatorname{TV}\left(u_{o}\right)+\operatorname{TV}\left(u_{b}\right)+\left|u_{b}(0+)-u_{o}(0+)\right| .
\end{aligned}
$$

The above estimates ensure that $u \in\left(\mathbf{L}^{\infty} \cap \mathbf{B V}\right)\left([0, T] \times \mathbb{R}_{+} ; \mathbb{R}\right)$.

Proof of Theorem 2.4. To exploit the semigroup notation as in [1, 5, 6], we assume without loss of generality that $T=+\infty$.

As in (4.8), define for any positive $\varepsilon$ the $\varepsilon$-approximate fluxes $f^{\varepsilon}, g^{\varepsilon} \in \mathbf{P L C}(\mathbb{R} ; \mathbb{R})$, the $\varepsilon$-approximate initial datum $u_{o}^{\varepsilon}$ and boundary datum $u_{b}^{\varepsilon}$. Let $\mathcal{D}^{\varepsilon}$ be the set of pairs $\mathbf{p}=$ $\left(u_{o}^{\varepsilon}, u_{b}^{\varepsilon}\right)$ such that $u_{o}^{\varepsilon} \in\left(\mathbf{L}^{1} \cap \mathbf{B V}\right)\left(\mathbb{R}_{+} ; \varepsilon \mathbb{Z}\right)$ and $u_{b}^{\varepsilon} \in\left(\mathbf{L}^{1} \cap \mathbf{B V}\right)\left(\mathbb{R}_{+} ; \varepsilon \mathbb{Z}\right)$, equipped with the norm $\left\|\left(u_{o}^{\varepsilon}, u_{b}^{\varepsilon}\right)\right\|_{\mathcal{D}^{\varepsilon}}=\max \left\{\left\|u_{o}^{\varepsilon}\right\|_{\mathbf{L}^{1}\left(\mathbb{R}^{+} ; \mathbb{R}\right)},\left\|u_{b}^{\varepsilon}\right\|_{\mathbf{L}^{1}\left(\mathbb{R}_{+} ; \mathbb{R}\right)}\right\}$. The algorithm used in the proof of Proposition 2.3 yields the semigroups

$$
\begin{aligned}
& \begin{array}{llllllllllllllll}
S^{\varepsilon} & : & \mathbb{R}_{+} & \times & \mathcal{D}^{\varepsilon} & \rightarrow & \mathcal{D}^{\varepsilon} \quad S^{g^{\varepsilon}} & : & \mathbb{R}_{+} & \times & \mathcal{D}^{\varepsilon} & \rightarrow & \mathcal{D}^{\varepsilon}
\end{array} \\
& t \quad, \quad\left(u_{o}^{\varepsilon}, u_{b}^{\varepsilon}\right) \mapsto\left(u^{\varepsilon}(t), \mathscr{T}_{t} u_{b}^{\varepsilon}\right) \quad t \quad, \quad\left(u_{o}^{\varepsilon}, u_{b}^{\varepsilon}\right) \mapsto\left(v^{\varepsilon}(t), \mathscr{T}_{t} u_{b}^{\varepsilon}\right)
\end{aligned}
$$

using the notation (2.3). Note that $t \rightarrow u^{\varepsilon}(t)$ and $t \rightarrow v^{\varepsilon}(t)$ are at the same time $\varepsilon^{-}$ approximate wave front tracking solutions to 2.9 and exact solutions to

$$
\left\{\begin{array} { l r l } 
{ \partial _ { t } u ^ { \varepsilon } + \partial _ { x } f ^ { \varepsilon } ( u ^ { \varepsilon } ) = 0 } & { ( t , x ) \in \mathbb { R } _ { + } \times \mathbb { R } _ { + } } \\
{ u ^ { \varepsilon } ( 0 , x ) = u _ { o } ^ { \varepsilon } ( x ) } & { x \in \mathbb { R } _ { + } } \\
{ u ^ { \varepsilon } ( t , 0 ) = u _ { b } ^ { \varepsilon } ( t ) } & { t \in \mathbb { R } _ { + } }
\end{array} \quad \text { and } \left\{\begin{array}{ll}
\partial_{t} v^{\varepsilon}+\partial_{x} g^{\varepsilon}\left(v^{\varepsilon}\right)=0 & (t, x) \in \mathbb{R}_{+} \times \mathbb{R}_{+} \\
v^{\varepsilon}(0, x)=u_{o}^{\varepsilon}(x) & x \in \mathbb{R}_{+} \\
v^{\varepsilon}(t, 0)=u_{b}^{\varepsilon}(t) & t \in \mathbb{R}_{+} .
\end{array}\right.\right.
$$

Hence, applying Proposition 2.2 and using the above choice of the norm in $\mathcal{D}^{\varepsilon}$, we have that $S_{t}^{f^{\varepsilon}}$ and $S_{t}^{g^{\varepsilon}}$ are Lipschitz continuous in both arguments, with

$$
\operatorname{Lip}\left(S_{t}^{g^{\varepsilon}}\right) \leq \max \left\{1,\left\|\left(g^{\varepsilon}\right)^{\prime}\right\|_{\mathbf{L}^{\infty}\left(\mathcal{U}_{t}^{\varepsilon} ; \mathbb{R}\right)}\right\} \leq \max \left\{1,\left\|g^{\prime}\right\|_{\mathbf{L}^{\infty}\left(\mathcal{U}_{t} ; \mathbb{R}\right)}\right\},
$$

where, using the notation 2.2 ,

$$
\mathcal{U}_{t}^{\varepsilon}=\mathcal{U}\left(u_{o}^{\varepsilon}, u_{b \mid[0, t]}^{\varepsilon}\right), \quad \mathcal{U}_{t}=\mathcal{U}\left(u_{o}, u_{b \mid[0, t]}\right) \quad \text { and } \quad \mathcal{U}_{t}^{\varepsilon} \subseteq \mathcal{U}_{t}
$$

due to (4.8). By [6, Theorem 2.9],

$$
\begin{aligned}
& \left\|u^{\varepsilon}(t)-v^{\varepsilon}(t)\right\|_{\mathbf{L}^{1}\left(\mathbb{R}_{+} ; \mathbb{R}\right)} \\
= & \left\|S_{t}^{f^{\varepsilon}}\left(u_{o}^{\varepsilon}, u_{b}^{\varepsilon}\right)-S_{t}^{g^{\varepsilon}}\left(u_{o}^{\varepsilon}, u_{b}^{\varepsilon}\right)\right\|_{\mathbf{L}^{1}\left(\mathbb{R}_{+} ; \mathbb{R}^{2}\right)} \\
\leq & \operatorname{Lip}\left(S_{t}^{g^{\varepsilon}}\right) \int_{0}^{t} \liminf _{h \rightarrow 0} \frac{1}{h}\left\|S_{h}^{g^{\varepsilon}} S_{\tau}^{f^{\varepsilon}}\left(u_{o}^{\varepsilon}, u_{b}^{\varepsilon}\right)-S_{h}^{f^{\varepsilon}} S_{\tau}^{f^{\varepsilon}}\left(u_{o}^{\varepsilon}, u_{b}^{\varepsilon}\right)\right\|_{\mathbf{L}^{1}\left(\mathbb{R}_{+} ; \mathbb{R}^{2}\right)} \mathrm{d} \tau .
\end{aligned}
$$

To simplify the notation, introduce $\left(w, w_{b}\right)=S_{\tau}^{f^{\varepsilon}}\left(u_{o}^{\varepsilon}, u_{b}^{\varepsilon}\right)$. Outside a finite set of times $\tau$, each Riemann problem for $f^{\varepsilon}$ in $w$ is solved by a single wave with speed $\lambda^{f}$. Let $\bar{x}$ be either 0 or a 


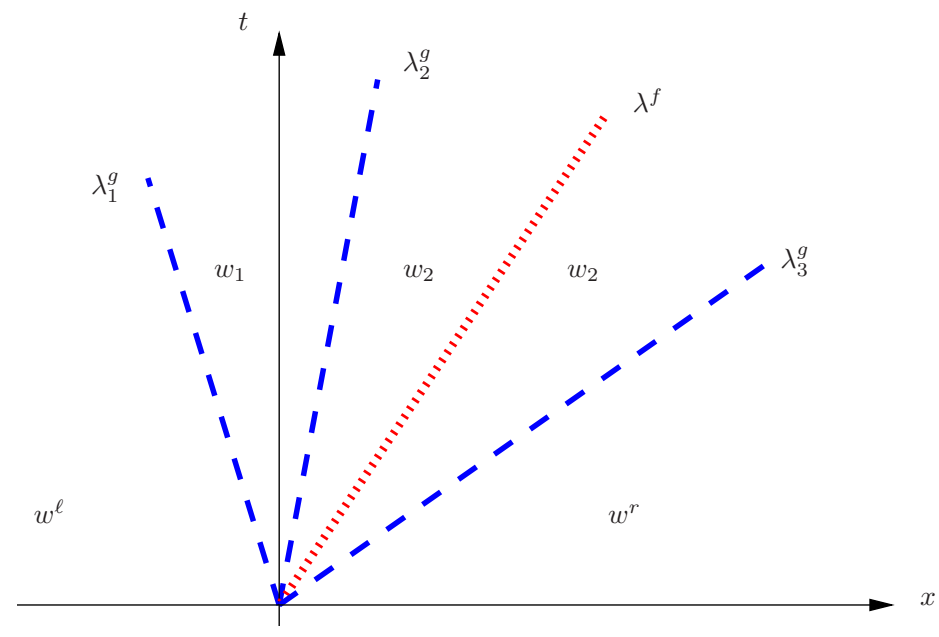

Figure 1: Notation used in the proof of Theorem 2.4 with $n^{\ell}=2$ and $n^{r}=1$.

point of jump in $w$. If $\bar{x}=0$, set $w^{\ell}=w_{b}(0+)=u_{b}^{\varepsilon}(\tau+)$, whereas $w^{\ell}=w(\bar{x}-)$ when $\bar{x}>0$. In both cases, let $w^{r}=w(\bar{x}+)$. In general, the solution to the Riemann problem for $g^{\varepsilon}$ with data $w^{\ell}$ and $w^{r}$ contains $n^{\ell}$ waves with speeds $\lambda_{1}^{g}<\cdots<\lambda_{n^{\ell}}^{g} \leq \lambda^{f}$ and $n^{r}$ waves with speeds $\lambda^{f}<\lambda_{n^{\ell}+1}^{g}<\cdots<\lambda_{n^{\ell}+n^{r}}^{g}$, see Figure 1. Assume that the intermediate states are increasing $w^{\ell}<w_{1}<\cdots<w_{n^{\ell}}<w_{n^{\ell}+1}<\ldots<w_{n^{\ell}+n^{r}}<w^{r}$, the other case being entirely analogous. For a sufficiently small $\delta>0$, call $I_{\delta}=[0, \delta]$ if $\bar{x}=0$ and $I_{\delta}=[\bar{x}-\delta, \bar{x}+\delta]$ if $\bar{x}>0$. We compute the integrand in (4.31) on $I_{\delta}$ through a repeated use of Rankine-Hugoniot condition:

$$
\begin{aligned}
& \frac{1}{h}\left\|S_{h}^{g^{\varepsilon}} S_{\tau}^{f^{\varepsilon}}\left(u_{o}^{\varepsilon}, u_{b}^{\varepsilon}\right)-S_{h}^{f^{\varepsilon}} S_{\tau}^{f^{\varepsilon}}\left(u_{o}^{\varepsilon}, u_{b}^{\varepsilon}\right)\right\|_{\mathbf{L}^{1}\left(I_{\delta} ; \mathbb{R}^{2}\right)} \\
= & \frac{1}{h}\left\|S_{h}^{g^{\varepsilon}}\left(w, w_{b}\right)-S_{h}^{f^{\varepsilon}}\left(w, w_{b}\right)\right\|_{\mathbf{L}^{1}\left(I_{\delta} ; \mathbb{R}^{2}\right)} \\
= & \sum_{i=1}^{n^{\ell}-1}\left|w_{i}-w^{\ell}\right|\left|\lambda_{i+1}^{g}-\lambda_{i}^{g}\right|+\left|w_{n^{\ell}}-w^{\ell}\right|\left|\lambda^{f}-\lambda_{n^{\ell}}^{g}\right| \\
& +\left|w^{r}-w_{n^{\ell}}\right|\left|\lambda_{n^{\ell}+1}^{g}-\lambda^{f}\right|+\sum_{i=1}^{n^{r}-1}\left|w^{r}-w_{n^{\ell}+i}\right|\left|\lambda_{n^{\ell}+i+1}^{g}-\lambda_{n^{\ell}+i}^{g}\right| \\
= & \sum_{i=1}^{n^{\ell}-1}\left(w_{i}-w^{\ell}\right)\left(\lambda_{i+1}^{g}-\lambda_{i}^{g}\right)+\left(w_{n^{\ell}}-w^{\ell}\right)\left(\lambda^{f}-\lambda_{n^{\ell}}^{g}\right) \\
& +\left(w^{r}-w_{n^{\ell}}\right)\left(\lambda_{n^{\ell}+1}^{g}-\lambda^{f}\right)+\sum_{i=1}^{n^{r}-1}\left(w^{r}-w_{n^{\ell}+i}\right)\left(\lambda_{n^{\ell}+i+1}^{g}-\lambda_{n^{\ell}+i}^{g}\right) \\
= & \left(g^{\varepsilon}\left(w^{r}\right)-g^{\varepsilon}\left(w_{n^{\ell}}\right)\right)-\left(g^{\varepsilon}\left(w_{n^{\ell}}\right)-g^{\varepsilon}\left(w^{\ell}\right)\right)+\left(w_{n^{\ell}}-w^{\ell}\right) \lambda^{f}-\left(w^{r}-w_{\left.n^{\ell}\right) \lambda^{f} .} .\right.
\end{aligned}
$$

Note that by Oleinik Entropy condition [9, Formula (8.4.3)]

$$
\frac{f^{\varepsilon}\left(w^{r}\right)-f^{\varepsilon}\left(w_{n^{\ell}}\right)}{w^{r}-w_{n^{\ell}}} \leq \lambda^{f} \leq \frac{f^{\varepsilon}\left(w_{n^{\ell}}\right)-f^{\varepsilon}\left(w^{\ell}\right)}{w_{n^{\ell}}-w^{\ell}},
$$

so that, using (4.8) and the fact that $w^{\ell}, w_{n^{\ell}}, w^{\ell} \in \varepsilon \mathbb{Z}$, continuing the estimate 4.32), we 
obtain:

$$
\begin{aligned}
& \frac{1}{h}\left\|S_{h}^{g^{\varepsilon}} S_{\tau}^{f^{\varepsilon}}\left(u_{o}^{\varepsilon}, u_{b}^{\varepsilon}\right)-S_{h}^{f^{\varepsilon}} S_{\tau}^{f^{\varepsilon}}\left(u_{o}^{\varepsilon}, u_{b}^{\varepsilon}\right)\right\|_{\mathbf{L}^{1}\left(I_{\delta} ; \mathbb{R}^{2}\right)} \\
\leq & \left(\left(g^{\varepsilon}-f^{\varepsilon}\right)\left(w^{r}\right)-\left(g^{\varepsilon}-f^{\varepsilon}\right)\left(w_{n^{\ell}}\right)\right)-\left(\left(g^{\varepsilon}-f^{\varepsilon}\right)\left(w_{n^{\ell}}\right)-\left(g^{\varepsilon}-f^{\varepsilon}\right)\left(w^{\ell}\right)\right) \\
= & \left((g-f)\left(w^{r}\right)-(g-f)\left(w_{\left.n^{\ell}\right)}\right)-\left((g-f)\left(w_{n^{\ell}}\right)-(g-f)\left(w^{\ell}\right)\right)\right. \\
\leq & \|D(g-f)\|_{\mathbf{C}^{0}\left(\left[w_{n^{\ell}}, w^{r}\right] ; \mathbb{R}\right)}\left|w^{r}-w_{n^{\ell}}\right|+\|D(g-f)\|_{\mathbf{C}^{0}\left(\left[w^{\ell}, w_{\left.\left.n^{\ell}\right] ; \mathbb{R}\right)}\left|w_{n^{\ell}}-w^{\ell}\right|\right.\right.}\left|w^{r}-w^{\ell}\right| . \\
\leq & \|D(g-f)\|_{\mathbf{C}^{0}\left(\left[w^{\ell}, w^{r}\right] ; \mathbb{R}\right)} \mid
\end{aligned}
$$

By $4.30, \mathcal{U}_{\tau}^{\varepsilon}=\mathcal{U}\left(u_{o}^{\varepsilon}, u_{b \mid[0, \tau]}^{\varepsilon}\right) \supseteq \mathcal{U}\left(w, w_{b}\right)$. Considering all Riemann problems for $u^{\varepsilon}$ at time $\tau$ along $\mathbb{R}_{+}$, the integrand in 4.31 becomes

$$
\begin{aligned}
& \frac{1}{h}\left\|S_{h}^{g^{\varepsilon}} S_{\tau}^{f^{\varepsilon}}\left(u_{o}^{\varepsilon}, u_{b}^{\varepsilon}\right)-S_{h}^{f^{\varepsilon}} S_{\tau}^{f^{\varepsilon}}\left(u_{o}^{\varepsilon}, u_{b}^{\varepsilon}\right)\right\|_{\mathbf{L}^{1}\left(\mathbb{R}_{+} ; \mathbb{R}^{2}\right)} \\
\leq & \|D(g-f)\|_{\mathbf{C}^{0}\left(\mathcal{U}_{\tau}^{\varepsilon} ; \mathbb{R}\right)}\left(\operatorname{TV}(w)+\left|w_{b}(0+)-w(0+)\right|\right) .
\end{aligned}
$$

Exploiting the functional $V^{\varepsilon}$ defined in $(4.13)$ and the fact that $V^{\varepsilon}(\tau) \leq V^{\varepsilon}(0)$, we obtain

$$
\begin{aligned}
& \frac{1}{h}\left\|S_{h}^{g^{\varepsilon}} S_{\tau}^{f^{\varepsilon}}\left(u_{o}^{\varepsilon}, u_{b}^{\varepsilon}\right)-S_{h}^{f^{\varepsilon}} S_{\tau}^{f^{\varepsilon}}\left(u_{o}^{\varepsilon}, u_{b}^{\varepsilon}\right)\right\|_{\mathbf{L}^{1}\left(\mathbb{R}_{+} ; \mathbb{R}^{2}\right)} \\
\leq & \|D(g-f)\|_{\mathbf{C}^{0}\left(\mathcal{U}_{\tau}^{\varepsilon} ; \mathbb{R}\right)}\left(\operatorname{TV}\left(u_{o}^{\varepsilon}\right)+\operatorname{TV}\left(u_{b}^{\varepsilon} ;[0, \tau]\right)+\left|u_{b}^{\varepsilon}(0+)-u_{o}^{\varepsilon}(0+)\right|\right) .
\end{aligned}
$$

Hence, 4.31 becomes

$$
\begin{aligned}
& \left\|u^{\varepsilon}(t)-v^{\varepsilon}(t)\right\|_{\mathbf{L}^{1}\left(\mathbb{R}_{+} ; \mathbb{R}\right)} \\
\leq & t \operatorname{Lip}\left(S_{t}^{g^{\varepsilon}}\right)\|D(g-f)\|_{\mathbf{C}^{0}\left(\mathcal{U}_{t}^{\varepsilon} ; \mathbb{R}\right)}\left(\operatorname{TV}\left(u_{o}^{\varepsilon}\right)+\operatorname{TV}\left(u_{b}^{\varepsilon} ;[0, t]\right)+\left|u_{b}^{\varepsilon}(0+)-u_{o}^{\varepsilon}(0+)\right|\right),
\end{aligned}
$$

where $\operatorname{Lip}\left(S_{t}^{g^{\varepsilon}}\right)$ is estimated as in 4.29 .

Let now $u$ and $v$ be the solutions to the problems $(2.9)$. Similarly to above, let $\mathcal{D}$ be the set of pairs $\mathbf{p}=\left(u_{o}, u_{b}\right)$ such that $u_{o} \in\left(\mathbf{L}^{1} \cap \mathbf{B V}\right)\left(\mathbb{R}_{+} ; \mathbb{R}\right)$ and $u_{b} \in\left(\mathbf{L}^{1} \cap \mathbf{B V}\right)\left(\mathbb{R}_{+} ; \mathbb{R}\right)$. Thanks to Proposition 2.3, the following two semigroups are then defined as limit of the semigroups $S^{f^{\varepsilon}}$ and $S^{g^{\varepsilon}}$ introduced above:

$$
\begin{aligned}
& \begin{array}{llllllllllllllllllll}
S^{f} & : & \mathbb{R}_{+} & \times & \mathcal{D} & \rightarrow & \mathcal{D} & S^{g} & : & \mathbb{R}_{+} & \times & \mathcal{D} & \rightarrow & \mathcal{D}
\end{array} \\
& t \quad, \quad\left(u_{o}, u_{b}\right) \mapsto\left(u(t), \mathscr{T}_{t} u_{b}\right) \quad t \quad, \quad\left(u_{o}, u_{b}\right) \mapsto\left(v(t), \mathscr{T}_{t} u_{b}\right) .
\end{aligned}
$$

Let $u_{o}^{\varepsilon}$ and $u_{b}^{\varepsilon}$ approximate $u_{o}$ and $u_{b}$ as in (4.8). Clearly $\left(u_{o}^{\varepsilon}, u_{b}^{\varepsilon}\right) \in \mathcal{D}^{\varepsilon}$. Compute

$$
\begin{aligned}
\|u(t)-v(t)\|_{\mathbf{L}^{1}\left(\mathbb{R}_{+} ; \mathbb{R}\right)}= & \left\|S_{t}^{f}\left(u_{o}, u_{b}\right)-S_{t}^{g}\left(u_{o}, u_{b}\right)\right\|_{\mathbf{L}^{1}\left(\mathbb{R}_{+} ; \mathbb{R}^{2}\right)} \\
\leq & \left\|S_{t}^{f}\left(u_{o}, u_{b}\right)-S_{t}^{f^{\varepsilon}}\left(u_{o}^{\varepsilon}, u_{b}^{\varepsilon}\right)\right\|_{\mathbf{L}^{1}\left(\mathbb{R}_{+} ; \mathbb{R}^{2}\right)} \\
& +\left\|S_{t}^{f^{\varepsilon}}\left(u_{o}^{\varepsilon}, u_{b}^{\varepsilon}\right)-S_{t}^{g^{\varepsilon}}\left(u_{o}^{\varepsilon}, u_{b}^{\varepsilon}\right)\right\|_{\mathbf{L}^{1}\left(\mathbb{R}_{+} ; \mathbb{R}^{2}\right)}
\end{aligned}
$$




$$
+\left\|S_{t}^{g^{\varepsilon}}\left(u_{o}^{\varepsilon}, u_{b}^{\varepsilon}\right)-S_{t}^{g}\left(u_{o}, u_{b}\right)\right\|_{\mathbf{L}^{1}\left(\mathbb{R}_{+} ; \mathbb{R}^{2}\right)} .
$$

Thanks to 4.8 and (4.33), the second addend (4.35) can be estimated as

$$
\begin{aligned}
& \left\|S_{t}^{f^{\varepsilon}}\left(u_{o}^{\varepsilon}, u_{b}^{\varepsilon}\right)-S_{t}^{g^{\varepsilon}}\left(u_{o}^{\varepsilon}, u_{b}^{\varepsilon}\right)\right\|_{\mathbf{L}^{1}\left(\mathbb{R}_{+} ; \mathbb{R}^{2}\right)} \\
\leq & t \operatorname{Lip}\left(S_{t}^{g^{\varepsilon}}\right)\|D(g-f)\|_{\mathbf{C}^{0}\left(\mathcal{U}_{t}^{\varepsilon} ; \mathbb{R}\right)}\left(\operatorname{TV}\left(u_{o}^{\varepsilon}\right)+\operatorname{TV}\left(u_{b}^{\varepsilon} ;[0, t]\right)+\left|u_{b}^{\varepsilon}(0+)-u_{o}^{\varepsilon}(0+)\right|\right) \\
\leq & t \max \left\{1,\left\|g^{\prime}\right\|_{\mathbf{L}^{\infty}\left(\mathcal{U}_{t} ; \mathbb{R}\right)}\right\}\|D(g-f)\|_{\mathbf{C}^{0}\left(\mathcal{U}_{t} ; \mathbb{R}\right)}\left[\operatorname{TV}\left(u_{o}\right)+\operatorname{TV}\left(u_{b} ;[0, t]\right)+\left|u_{b}(0+)-u_{o}(0+)\right|\right]
\end{aligned}
$$

where we used (4.29) and (4.30). The terms (4.34) and (4.36) converge to 0 as $\varepsilon \rightarrow 0$, due to the construction of the $\varepsilon$-solutions above. The proof is completed.

\subsection{Proofs related to the Non Autonomous IBVP on the Half-Line}

Proof of Proposition 2.5. The proof consists of several steps.

N.1) Construction of the Approximate Solutions. For $n \in \mathbb{N}$ and $i=0, \ldots, 2^{n}$, define $T_{n}^{i}=\frac{i}{2^{n}} T$. For $i=0, \ldots, 2^{n}-1$, we recursively consider the autonomous problems

$$
\left\{\begin{array}{lc}
\partial_{t} u_{n}^{i}+\partial_{x} f\left(T_{n}^{i}, u_{n}^{i}\right)=0 & (t, x) \in\left[T_{n}^{i}, T_{n}^{i+1}\right] \times \mathbb{R}_{+} \\
u_{n}^{i}\left(T_{n}^{i}, x\right)=u_{n}^{i-1}\left(T_{n}^{i}, x\right) & x \in \mathbb{R}_{+} \\
u_{n}^{i}(t, 0)=u_{b}(t) & t \in\left[T_{n}^{i}, T_{n}^{i+1}\right],
\end{array}\right.
$$

where we set $u_{n}^{-1}=u_{o}$. Each of these problems falls within the scope of Proposition 2.3 . Therefore, for any $\varepsilon>0$ define as in 4.8 the $\varepsilon$-approximate initial and boundary data $u_{o}^{\varepsilon}$ and $u_{b}^{\varepsilon}$. Moreover, for $i=0, \ldots, 2^{n}-1$, define the $\varepsilon$-approximate fluxes $u \rightarrow f^{\varepsilon}\left(T_{n}^{i}, u\right)$. Call $u_{n}^{i, \varepsilon}$ the wave front tracking $\varepsilon$-approximate solution to 4.37 constructed as in Proposition 2.3 . Then, the solution $u_{n}^{i}$ to 4.37) satisfies $u_{n}^{i}=\lim _{\varepsilon \rightarrow 0} u_{n}^{i, \varepsilon}$.

For $i=0, \ldots, 2^{n}-1$ define

$$
\begin{aligned}
u_{n}(t) & =u_{n}^{i}(t) \quad \text { for } \quad t \in\left[T_{n}^{i}, T_{n}^{i+1}\right] \\
V_{n}^{i, \varepsilon} & =\operatorname{TV}\left(u_{n}^{i-1, \varepsilon}\left(T_{n}^{i}\right)\right)+\operatorname{TV}\left(u_{b}^{\varepsilon} ;\left[T_{n}^{i}, T\right]\right)+\left|u_{b}^{\varepsilon}\left(T_{n}^{i}+\right)-u_{n}^{i-1, \varepsilon}\left(T_{n}^{i}, 0+\right)\right| \\
\mathcal{U}_{t} & \left.=\mathcal{U}\left(u_{o}, u_{b \mid[0, t]}\right) \quad \text { and } \quad \mathcal{U}=\mathcal{U}_{T} \quad \text { with the notation } 2.2\right] \\
L & =1+\left\|\partial_{u} f\right\|_{\mathbf{L}^{\infty}([0, T] \times \mathcal{U} ; \mathbb{R})} \\
K & =\operatorname{TV}\left(u_{o}\right)+\operatorname{TV}\left(u_{b} ;[0, T]\right)+\left|u_{b}(0+)-u_{o}(0+)\right| .
\end{aligned}
$$

The quantity $V_{n}^{i, \varepsilon}$ is the functional defined in 4.13 computed at time $t=T_{n}^{i}$. Hence, by $\mathbf{A . 3}$ in the proof of Proposition 2.3 , we recursively obtain

$$
V_{n}^{i, \varepsilon} \leq V_{n}^{i-1, \varepsilon} \quad \text { for all } \quad i=1, \ldots, 2^{n}-1 .
$$



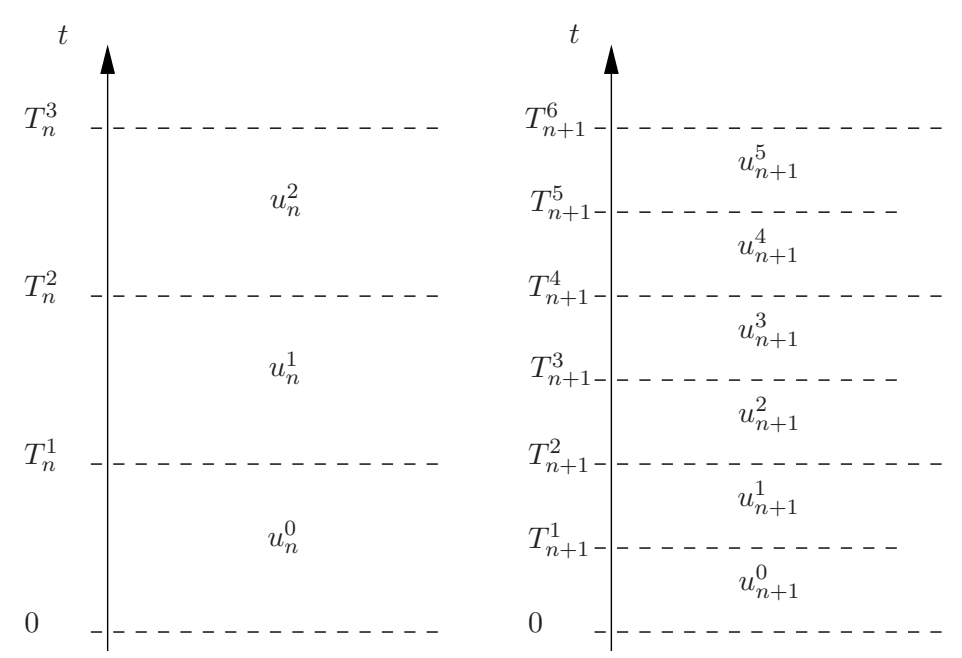

Figure 2: Relations between the time interval $\left[T_{n}^{i}, T_{n}^{i+1}\right]$, where the approximate solution is $u_{n}^{i}$, and the time intervals $\left[T_{n+1}^{2 i}, T_{n+1}^{2 i+1}\right]$ and $\left[T_{n+1}^{2 i+1}, T_{n+1}^{2 i+2}\right]$, where the approximate solutions are $u_{n+1}^{2 i}$ and $u_{n+1}^{2 i+1}$, see (4.37).

N.2) $\boldsymbol{u}_{\boldsymbol{n}}$ is a Cauchy sequence in $\mathbf{C}^{0}\left([0, T] ; \mathbf{L}^{1}\left(\mathbb{R}_{+} ; \mathbb{R}\right)\right)$. Here and in what follows, we use the norm $\|u\|_{\mathbf{C}^{0}\left([0, T] ; \mathbf{L}^{1}\left(\mathbb{R}_{+} ; \mathbb{R}\right)\right)}=\sup _{t \in[0, T]}\|u(t)\|_{\mathbf{L}^{1}\left(\mathbb{R}_{+} ; \mathbb{R}\right)}$. It is sufficient to obtain

$$
\left\|u_{n+1}-u_{n}\right\|_{\mathbf{C}^{0}\left([0, T] ; \mathbf{L}^{1}\left(\mathbb{R}_{+} ; \mathbb{R}\right)\right)}=\mathcal{O}(1) 2^{-n}
$$

as soon as the constant $\mathcal{O}(1)$ is independent of $n$, which in turn follows from the bounds

$$
\begin{array}{ll}
\left\|u_{n+1}^{2 j}(t)-u_{n}^{j}(t)\right\|_{\mathbf{L}^{1}\left(\mathbb{R}_{+} ; \mathbb{R}\right)} \leq j L K\left\|\partial_{t} \partial_{u} f\right\|_{\mathbf{L}^{\infty}([0, T] \times \mathcal{U} ; \mathbb{R})}\left(\frac{T}{2^{n+1}}\right)^{2} & t \in\left[T_{n}^{j}, T_{n+1}^{2 j+1}\right] \\
\left\|u_{n+1}^{2 j+1}(t)-u_{n}^{j}(t)\right\|_{\mathbf{L}^{1}\left(\mathbb{R}_{+} ; \mathbb{R}\right)} \leq(j+1) L K\left\|\partial_{t} \partial_{u} f\right\|_{\mathbf{L}^{\infty}([0, T] \times \mathcal{U} ; \mathbb{R})}\left(\frac{T}{2^{n+1}}\right)^{2} & t \in\left[T_{n+1}^{2 j+1}, T_{n}^{j+1}\right] .
\end{array}
$$

Fix $n$ and proceed inductively on $j$.

$\star j=0$. Assume first that $t \in\left[0, T_{n+1}^{1}\right]$, see Figure 2. By 4.37) we immediately have $u_{n+1}^{0}(t)=u_{n}^{0}(t)$ for $t \in\left[0, T_{n+1}^{1}\right]$. Let now $t \in\left[T_{n+1}^{1}, T_{n}^{1}\right]$, see Figure 2 . Compute

$$
\begin{aligned}
& \left\|u_{n+1}^{1}(t)-u_{n}^{0}(t)\right\|_{\mathbf{L}^{1}\left(\mathbb{R}_{+} ; \mathbb{R}\right)}+\left\|u_{n+1}^{1, \varepsilon}(t)-u_{n}^{0, \varepsilon}(t)\right\|_{\mathbf{L}^{1}\left(\mathbb{R}_{+} ; \mathbb{R}\right)}+\left\|u_{n}^{0, \varepsilon}(t)-u_{n}^{0}(t)\right\|_{\mathbf{L}^{1}\left(\mathbb{R}_{+} ; \mathbb{R}\right)} \\
\leq & \left\|u_{n+1}^{1}(t)-u_{n+1}^{1, \varepsilon}(t)\right\|_{\mathbf{L}^{1}\left(\mathbb{R}_{+} ; \mathbb{R}\right)}
\end{aligned}
$$

Focus on the term in the middle: an application of 4.33, yields

$$
\begin{aligned}
& \left\|u_{n+1}^{1, \varepsilon}(t)-u_{n}^{0, \varepsilon}(t)\right\|_{\mathbf{L}^{1}\left(\mathbb{R}_{+} ; \mathbb{R}\right)} \\
\leq & L \sup _{u \in \mathcal{U}}\left|\partial_{u} f^{\varepsilon}\left(T_{n+1}^{1}, u\right)-\partial_{u} f^{\varepsilon}(0, u)\right|\left(t-T_{n+1}^{1}\right) \\
& \times\left(\operatorname{TV}\left(u_{n+1}^{0, \varepsilon}\left(T_{n+1}^{1}\right)\right)+\operatorname{TV}\left(u_{b}^{\varepsilon} ;\left[T_{n+1}^{1}, t\right]\right)+\left|u_{b}^{\varepsilon}\left(T_{n+1}^{1}+\right)-u_{n+1}^{0, \varepsilon}\left(T_{n+1}^{1}, 0+\right)\right|\right) \\
\leq & L\left\|\partial_{t} \partial_{u} f\right\|_{\mathbf{L}^{\infty}([0, T] \times \mathcal{U} ; \mathbb{R})}\left(\frac{T}{2^{n+1}}\right)^{2}\left(V_{n+1}^{1, \varepsilon}-\operatorname{TV}\left(u_{b}^{\varepsilon} ;[t, T]\right)\right)
\end{aligned}
$$




$$
\begin{aligned}
& \leq L\left\|\partial_{t} \partial_{u} f\right\|_{\mathbf{L}^{\infty}([0, T] \times \mathcal{U} ; \mathbb{R})}\left(\frac{T}{2^{n+1}}\right)^{2}\left(V_{n+1}^{0, \varepsilon}-\operatorname{TV}\left(u_{b}^{\varepsilon} ;[t, T]\right)\right) \\
& \leq L\left\|\partial_{t} \partial_{u} f\right\|_{\mathbf{L}^{\infty}([0, T] \times \mathcal{U} ; \mathbb{R})}\left(\frac{T}{2^{n+1}}\right)^{2}\left(\operatorname{TV}\left(u_{o}^{\varepsilon}\right)+\operatorname{TV}\left(u_{b}^{\varepsilon} ;[0, t]\right)+\left|u_{b}^{\varepsilon}(0+)-u_{o}^{\varepsilon}(0+)\right|\right) \\
& \leq L\left\|\partial_{t} \partial_{u} f\right\|_{\mathbf{L}^{\infty}([0, T] \times \mathcal{U} ; \mathbb{R})}\left(\frac{T}{2^{n+1}}\right)^{2}\left(\operatorname{TV}\left(u_{o}\right)+\operatorname{TV}\left(u_{b} ;[0, t]\right)+\left|u_{b}(0+)-u_{o}(0+)\right|\right) \\
& \leq L K\left\|\partial_{t} \partial_{u} f\right\|_{\mathbf{L}^{\infty}([0, T] \times \mathcal{U} ; \mathbb{R})}\left(\frac{T}{2^{n+1}}\right)^{2},
\end{aligned}
$$

where we used (4.40) and (4.8). Inserting the above estimate in (4.43) and letting $\varepsilon \rightarrow 0$ yield the desired result.

$\star j=1$. Assume first $t \in\left[T_{n}^{1}, T_{n+1}^{3}\right]$, see Figure 2. In this time interval, the $n$ - and the $(n+1)$-problem have the same flux, since $T_{n}^{1}=T_{n+1}^{2}$. An application of Proposition 2.2 to the autonomous problem (4.37) and using the result in the previous step $j=0$,

$$
\begin{aligned}
& \left\|u_{n+1}^{2}(t)-u_{n}^{1}(t)\right\|_{\mathbf{L}^{1}\left(\mathbb{R}_{+} ; \mathbb{R}\right)} \\
\leq & \left\|u_{n+1}^{1}\left(T_{n}^{1}\right)-u_{n}^{0}\left(T_{n}^{1}\right)\right\|_{\mathbf{L}^{1}\left(\mathbb{R}_{+} ; \mathbb{R}\right)} \\
\leq & L\left\|\partial_{t} \partial_{u} f\right\|_{\mathbf{L}^{\infty}([0, T] \times \mathcal{U} ; \mathbb{R})}\left(\frac{T}{2^{n+1}}\right)^{2}\left(\mathrm{TV}\left(u_{o}\right)+\mathrm{TV}\left(u_{b} ;\left[0, T_{n}^{1}\right]\right)+\left|u_{b}(0+)-u_{o}(0+)\right|\right) \\
\leq & L K\left\|\partial_{t} \partial_{u} f\right\|_{\mathbf{L}^{\infty}([0, T] \times \mathcal{U} ; \mathbb{R})}\left(\frac{T}{2^{n+1}}\right)^{2} .
\end{aligned}
$$

Let now $t \in\left[T_{n+1}^{3}, T_{n}^{2}\right]$, see Figure 2. Compute

$$
\begin{aligned}
& \left\|u_{n+1}^{3}(t)-u_{n}^{1}(t)\right\|_{\mathbf{L}^{1}\left(\mathbb{R}_{+} ; \mathbb{R}\right)} \\
\leq & \left\|u_{n+1}^{3}(t)-u_{n+1}^{3, \varepsilon}(t)\right\|_{\mathbf{L}^{1}\left(\mathbb{R}_{+} ; \mathbb{R}\right)}+\left\|u_{n+1}^{3, \varepsilon}(t)-u_{n}^{1, \varepsilon}(t)\right\|_{\mathbf{L}^{1}\left(\mathbb{R}_{+} ; \mathbb{R}\right)}+\left\|u_{n}^{1, \varepsilon}(t)-u_{n}^{1}(t)\right\|_{\mathbf{L}^{1}\left(\mathbb{R}_{+} ; \mathbb{R}\right)} .
\end{aligned}
$$

Focus on the term in the middle: an application of Proposition 2.2 and of 4.33 yields

$$
\begin{aligned}
& \left\|u_{n+1}^{3, \varepsilon}(t)-u_{n}^{1, \varepsilon}(t)\right\|_{\mathbf{L}^{1}\left(\mathbb{R}_{+} ; \mathbb{R}\right)} \\
\leq & \left\|u_{n+1}^{2, \varepsilon}\left(T_{n+1}^{3}\right)-u_{n}^{1, \varepsilon}\left(T_{n+1}^{3}\right)\right\|_{\mathbf{L}^{1}\left(\mathbb{R}_{+} ; \mathbb{R}\right)} \\
& +L \sup _{u \in \mathcal{U}}\left|\partial_{u} f^{\varepsilon}\left(T_{n+1}^{3}, u\right)-\partial_{u} f^{\varepsilon}\left(T_{n}^{1}, u\right)\right|\left(t-T_{n+1}^{3}\right) \\
& \quad \times\left(\operatorname{TV}\left(u_{n+1}^{2, \varepsilon}\left(T_{n+1}^{3}\right)\right)+\operatorname{TV}\left(u_{b}^{\varepsilon} ;\left[T_{n+1}^{3}, t\right]\right)+\left|u_{b}^{\varepsilon}\left(T_{n+1}^{3}+\right)-u_{n+1}^{2, \varepsilon}\left(T_{n+1}^{3}, 0+\right)\right|\right) .
\end{aligned}
$$

The term in the latter line above is estimated through a recursive use of 4.40 :

$$
\begin{aligned}
& \operatorname{TV}\left(u_{n+1}^{2, \varepsilon}\left(T_{n+1}^{3}\right)\right)+\operatorname{TV}\left(u_{b}^{\varepsilon} ;\left[T_{n+1}^{3}, t\right]\right)+\left|u_{b}^{\varepsilon}\left(T_{n+1}^{3}+\right)-u_{n+1}^{2, \varepsilon}\left(T_{n+1}^{3}, 0+\right)\right| \\
= & V_{n+1}^{3, \varepsilon}-\operatorname{TV}\left(u_{b}^{\varepsilon} ;[t, T]\right) \\
\leq & \operatorname{TV}\left(u_{o}^{\varepsilon}\right)+\operatorname{TV}\left(u_{b}^{\varepsilon}\right)+\left|u_{b}^{\varepsilon}(0+)-u_{o}^{\varepsilon}(0+)\right|-\operatorname{TV}\left(u_{b}^{\varepsilon} ;[t, T]\right)
\end{aligned}
$$




$$
\begin{aligned}
& \leq \mathrm{TV}\left(u_{o}\right)+\mathrm{TV}\left(u_{b},[0, t]\right)+\left|u_{b}(0+)-u_{o}(0+)\right| \\
& \leq K
\end{aligned}
$$

where we exploit 4.8). Hence, we recursively continue the estimate of 4.45):

$$
\begin{aligned}
& \left\|u_{n+1}^{3, \varepsilon}(t)-u_{n}^{1, \varepsilon}(t)\right\|_{\mathbf{L}^{1}\left(\mathbb{R}_{+} ; \mathbb{R}\right)} \\
\leq & \left\|u_{n+1}^{2, \varepsilon}\left(T_{n+1}^{3}\right)-u_{n}^{1, \varepsilon}\left(T_{n+1}^{3}\right)\right\|_{\mathbf{L}^{1}(\mathbb{R} ; ; \mathbb{R})}+L K \sup _{u \in \mathcal{U}}\left|\partial_{u} f\left(T_{n+1}^{3}, u\right)-\partial_{u} f\left(T_{n}^{1}, u\right)\right|\left(t-T_{n+1}^{3}\right) \\
\leq & 2 L K\left\|\partial_{t} \partial_{u} f\right\|_{\mathbf{L}^{\infty}([0, T] \times \mathcal{U} ; \mathbb{R})}\left(\frac{T}{2^{n+1}}\right)^{2} .
\end{aligned}
$$

Inserting the above estimate in (4.44) and letting $\varepsilon \rightarrow 0$ yield the desired result.

$\star j>1$. Assume first that $t \in\left[T_{n}^{j}, T_{n+1}^{2 j+1}\right]$. An application of Proposition 2.2 to 4.37) and the inductive hypothesis yield

$$
\begin{aligned}
\left\|u_{n+1}^{2 j}(t)-u_{n}^{j}(t)\right\|_{\mathbf{L}^{1}\left(\mathbb{R}_{+} ; \mathbb{R}\right)} & \leq\left\|u_{n+1}^{2 j-1}\left(T_{n}^{j}\right)-u_{n}^{j-1}\left(T_{n}^{j}\right)\right\|_{\mathbf{L}^{1}\left(\mathbb{R}_{+} ; \mathbb{R}\right)} \\
& \leq j L K\left\|\partial_{t} \partial_{u} f\right\|_{\mathbf{L}^{\infty}([0, T] \times \mathcal{U} ; \mathbb{R})}\left(\frac{T}{2^{n+1}}\right)^{2} .
\end{aligned}
$$

Let now $t \in\left[T_{n+1}^{2 j+1}, T_{n}^{j+1}\right]$. Compute

$$
\begin{aligned}
& \left\|u_{n+1}^{2 j+1}(t)-u_{n}^{j}(t)\right\|_{\mathbf{L}^{1}\left(\mathbb{R}_{+} ; \mathbb{R}\right)} \\
\leq & \left\|u_{n+1}^{2 j+1}(t)-u_{n+1}^{2 j+1, \varepsilon}(t)\right\|_{\mathbf{L}^{1}\left(\mathbb{R}_{+} ; \mathbb{R}\right)}+\left\|u_{n+1}^{2 j+1, \varepsilon}(t)-u_{n}^{j, \varepsilon}(t)\right\|_{\mathbf{L}^{1}\left(\mathbb{R}_{+} ; \mathbb{R}\right)}+\left\|u_{n}^{j, \varepsilon}(t)-u_{n}^{j}(t)\right\|_{\mathbf{L}^{1}\left(\mathbb{R}_{+} ; \mathbb{R}\right)}
\end{aligned}
$$

An application of Proposition 2.2 and of 4.33 to the term in the middle yields

$$
\begin{aligned}
& \\
& \quad\left\|u_{n+1}^{2 j+1, \varepsilon}(t)-u_{n}^{j, \varepsilon}(t)\right\|_{\mathbf{L}^{1}\left(\mathbb{R}_{+} ; \mathbb{R}\right)} \\
& \leq\left\|u_{n+1}^{2 j, \varepsilon}\left(T_{n+1}^{2 j+1}\right)-u_{n}^{j, \varepsilon}\left(T_{n+1}^{2 j+1}\right)\right\|_{\mathbf{L}^{1}\left(\mathbb{R}_{+} ; \mathbb{R}\right)} \\
& \quad+L \sup _{u \in \mathcal{U}}\left|\partial_{u} f^{\varepsilon}\left(T_{n+1}^{2 j+1}, u\right)-\partial_{u} f^{\varepsilon}\left(T_{n}^{j}, u\right)\right|\left(t-T_{n+1}^{2 j+1}\right) \\
& \quad \times\left(\operatorname{TV}\left(u_{n+1}^{2 j, \varepsilon}\left(T_{n+1}^{2 j+1}\right)\right)+\operatorname{TV}\left(u_{b}^{\varepsilon} ;\left[T_{n+1}^{2 j+1}, t\right]\right)+\left|u_{b}^{\varepsilon}\left(T_{n+1}^{2 j+1}+\right)-u_{n+1}^{2 j, \varepsilon}\left(T_{n+1}^{2 j+1}, 0+\right)\right|\right) .
\end{aligned}
$$

The term in the latter line above can be estimated thanks to 4.40 and (4.8)

$$
\begin{aligned}
& \operatorname{TV}\left(u_{n+1}^{2 j, \varepsilon}\left(T_{n+1}^{2 j+1}\right)\right)+\operatorname{TV}\left(u_{b}^{\varepsilon} ;\left[T_{n+1}^{2 j+1}, t\right]\right)+\left|u_{b}^{\varepsilon}\left(T_{n+1}^{2 j+1}+\right)-u_{n+1}^{2 j, \varepsilon}\left(T_{n+1}^{2 j+1}, 0+\right)\right| \\
= & V_{n+1}^{2 j+1, \varepsilon}-\operatorname{TV}\left(u_{b}^{\varepsilon} ;[t, T]\right) \\
\leq & \cdots \\
\leq & \operatorname{TV}\left(u_{o}^{\varepsilon}\right)+\operatorname{TV}\left(u_{b}^{\varepsilon}\right)+\left|u_{b}^{\varepsilon}(0+)-u_{o}^{\varepsilon}(0+)\right|-\operatorname{TV}\left(u_{b}^{\varepsilon} ;[t, T]\right) \\
\leq & \operatorname{TV}\left(u_{o}\right)+\operatorname{TV}\left(u_{b},[0, t]\right)+\left|u_{b}(0+)-u_{o}(0+)\right| \\
\leq & K .
\end{aligned}
$$


Hence, we continue the estimate of (4.47):

$$
\begin{aligned}
& \left\|u_{n+1}^{2 j+1, \varepsilon}(t)-u_{n}^{j, \varepsilon}(t)\right\|_{\mathbf{L}^{1}\left(\mathbb{R}_{+} ; \mathbb{R}\right)} \\
\leq & \left\|u_{n+1}^{2 j, \varepsilon}\left(T_{n+1}^{2 j+1}\right)-u_{n}^{j, \varepsilon}\left(T_{n+1}^{2 j+1}\right)\right\|_{\mathbf{L}^{1}\left(\mathbb{R}_{+} ; \mathbb{R}\right)}+L K \sup _{u \in \mathcal{U}}\left|\partial_{u} f^{\varepsilon}\left(T_{n+1}^{2 j+1}, u\right)-\partial_{u} f^{\varepsilon}\left(T_{n}^{j}, u\right)\right|\left(t-T_{n+1}^{2 j+1}\right) \\
\leq & (j+1) L K\left\|\partial_{t} \partial_{u} f\right\|_{\mathbf{L}^{\infty}([0, T] \times \mathcal{U} ; \mathbb{R})}\left(\frac{T}{2^{n+1}}\right)^{2},
\end{aligned}
$$

which inserted in (4.46) yields the desired result when passing to the limit $\varepsilon \rightarrow 0$.

This proves 4.42, obtaining 4.41 with $\mathcal{O}(1)=\frac{1}{4} L K\left\|\partial_{t} \partial_{u} f\right\|_{\mathbf{L}^{\infty}([0, T] \times \mathcal{U} ; \mathbb{R})} T^{2}$, so that $u_{n}$ is a Cauchy sequence in $\mathbf{C}^{0}\left([0, T] ; \mathbf{L}^{1}\left(\mathbb{R}_{+} ; \mathbb{R}\right)\right)$ : call $u$ its limit.

N.3) $\mathbf{L}^{\infty}$-Estimate. Observe moreover that for any $t \in[0, T]$, Point 2. in Proposition 2.3 implies that $\left\|u_{n}^{i}(t)\right\|_{\mathbf{L}^{\infty}\left(\mathbb{R}_{+} ; \mathbb{R}\right)} \leq \max \left\{\left\|u_{n}^{i-1}\left(T_{n}^{i}\right)\right\|_{\mathbf{L}^{\infty}\left(\mathbb{R}_{+} ; \mathbb{R}\right)},\left\|u_{b}\right\|_{\mathbf{L}^{\infty}\left(\left[T_{n}^{i}, t\right] ; \mathbb{R}\right)}\right\}$ for $i=$ $0, \ldots, 2^{n}-1$, and this recursively yields

$$
\left\|u_{n}(t)\right\|_{\mathbf{L}^{\infty}\left(\mathbb{R}_{+} ; \mathbb{R}\right)} \leq \max \left\{\left\|u_{o}\right\|_{\mathbf{L}^{\infty}\left(\mathbb{R}_{+} ; \mathbb{R}\right)},\left\|u_{b}\right\|_{\mathbf{L}^{\infty}([0, t] ; \mathbb{R})}\right\}
$$

which, in the limit $n \rightarrow+\infty$, gives Point 1 .

N.4) $u$ is a Weak Entropy Solution to (1.1). For any $\varphi \in \mathbf{C}_{c}^{1}\left(\mathbb{R} \times \mathbb{R} ; \mathbb{R}_{+}\right)$and any $k \in \mathbb{R}$, since each $u_{n}^{i}$ is a solution to 4.37 in the sense of Definition 2.1.

$$
\begin{aligned}
0 \leq & \int_{0}^{T} \int_{\mathbb{R}_{+}} \eta_{k}^{ \pm}\left(u_{n}(t, x)\right) \partial_{t} \varphi(t, x) \mathrm{d} x \mathrm{~d} t \\
& +\sum_{i=0}^{2^{n}-1} \int_{T_{n}^{i}}^{T_{n}^{i+1}} \int_{\mathbb{R}_{+}} \Phi_{k}^{ \pm}\left(T_{n}^{i}, u_{n}^{i}(t, x)\right) \partial_{x} \varphi(t, x) \mathrm{d} x \mathrm{~d} t \\
& +\int_{\mathbb{R}_{+}} \eta_{k}^{ \pm}\left(u_{o}(x)\right) \varphi(0, x) \mathrm{d} x-\int_{\mathbb{R}_{+}} \eta_{k}^{ \pm}\left(u_{n}(T, x)\right) \varphi(T, x) \mathrm{d} x \\
& +\sum_{i=0}^{2^{n}-1}\left\|\partial_{u} f\left(T_{n}^{i}, \cdot\right)\right\|_{\mathbf{L}^{\infty}(\mathcal{U} ; \mathbb{R})} \int_{T_{n}^{i}}^{T_{n}^{i+1}} \eta_{k}^{ \pm}\left(u_{b}(t)\right) \varphi(t, 0) \mathrm{d} t,
\end{aligned}
$$

with $\eta_{k}^{ \pm}$and $\Phi_{k}^{ \pm}$as in $(2.5)$. We compute the limit as $n \rightarrow+\infty$ of the lines above separately.

Since $\eta_{k}^{ \pm}$are Lipschitz continuous functions with Lipschitz constant 1, we obtain

$$
\begin{aligned}
{[4.49] \leq } & \int_{0}^{T} \int_{\mathbb{R}_{+}} \eta_{k}^{ \pm}(u(t, x)) \partial_{t} \varphi(t, x) \mathrm{d} x \mathrm{~d} t \\
& +\int_{0}^{T} \int_{\mathbb{R}_{+}}\left(\eta_{k}^{ \pm}\left(u_{n}(t, x)\right)-\eta_{k}^{ \pm}(u(t, x))\right) \partial_{t} \varphi(t, x) \mathrm{d} x \mathrm{~d} t \\
\leq & \int_{0}^{T} \int_{\mathbb{R}_{+}} \eta_{k}^{ \pm}(u(t, x)) \partial_{t} \varphi(t, x) \mathrm{d} x \mathrm{~d} t+\int_{0}^{T} \int_{\mathbb{R}_{+}}\left|u_{n}(t, x)-u(t, x)\right| \partial_{t} \varphi(t, x) \mathrm{d} x \mathrm{~d} t,
\end{aligned}
$$


and in the limit $n \rightarrow+\infty$ we get

$$
\lim _{n \rightarrow+\infty}[4.49]=\int_{0}^{T} \int_{\mathbb{R}_{+}} \eta_{k}^{ \pm}(u(t, x)) \partial_{t} \varphi(t, x) \mathrm{d} x \mathrm{~d} t .
$$

Concerning 4.50, compute

$$
\begin{aligned}
\Phi_{k}^{ \pm}\left(T_{n}^{i}, u_{n}^{i}(t, x)\right)= & \Phi_{k}^{ \pm}(t, u(t, x))+\left(\Phi_{k}^{ \pm}\left(T_{n}^{i}, u(t, x)\right)-\Phi_{k}^{ \pm}(t, u(t, x))\right) \\
& +\left(\Phi_{k}^{ \pm}\left(T_{n}^{i}, u_{n}^{i}(t, x)\right)-\Phi_{k}^{ \pm}\left(T_{n}^{i}, u(t, x)\right)\right) .
\end{aligned}
$$

To estimate the second term above, introduce the set $\mathcal{U}_{k}=\mathcal{U}\left(u_{o}, u_{b \mid[0, t]}, k\right)$ and compute

$$
\begin{aligned}
& \Phi_{k}^{ \pm}\left(T_{n}^{i}, u(t, x)\right)-\Phi_{k}^{ \pm}(t, u(t, x)) \\
= & \operatorname{sgn}^{ \pm}(u(t, x)-k)\left(f\left(T_{n}^{i}, u(t, x)\right)-f\left(T_{n}^{i}, k\right)-f(t, u(t, x))+f(t, k)\right) \\
\leq & \left\|\partial_{t} \partial_{u} f\right\|_{\mathbf{L}^{\infty}\left([0, t] \times \mathcal{U}_{k} ; \mathbb{R}\right)}|u(t, x)-k|\left|t-T_{n}^{i}\right| \\
\leq & \left\|\partial_{t} \partial_{u} f\right\|_{\mathbf{L}^{\infty}\left([0, t] \times \mathcal{U}_{k} ; \mathbb{R}\right)} \operatorname{diam}\left(\mathcal{U}_{k}\right) \frac{T}{2^{n}},
\end{aligned}
$$

so that

$$
\begin{array}{r}
\sum_{i=0}^{2^{n}-1} \int_{T_{n}^{i}}^{T_{n}^{i+1}} \int_{\mathbb{R}_{+}}\left(\Phi_{k}^{ \pm}\left(T_{n}^{i}, u(t, x)\right)-\Phi_{k}^{ \pm}(t, u(t, x))\right)\left|\partial_{x} \varphi(t, x)\right| \mathrm{d} x \mathrm{~d} t \\
\leq\left\|\partial_{t} \partial_{u} f\right\|_{\mathbf{L}^{\infty}\left([0, t] \times \mathcal{U}_{k} ; \mathbb{R}\right)} \operatorname{diam}\left(\mathcal{U}_{k}\right) \int_{\mathbb{R}_{+}} \sup _{t \in \mathbb{R}^{+}}\left|\partial_{x} \varphi(t, x)\right| \mathrm{d} x \sum_{i=0}^{2^{n}-1}\left(\frac{T}{2^{n}}\right)^{2},
\end{array}
$$

which clearly vanishes in the limit $n \rightarrow+\infty$.

To estimate the third term, observe that the maps $\Phi_{k}^{ \pm}$are Lipschitz continuous, see [14, Lemma 3], with Lipschitz constant $\left\|\partial_{u} f\right\|_{\mathbf{L}^{\infty}\left([0, t] \times \mathcal{U}_{t} ; \mathbb{R}\right)}$, so that

$$
\Phi_{k}^{ \pm}\left(T_{n}^{i}, u_{n}^{i}(t, x)\right)-\Phi_{k}^{ \pm}\left(T_{n}^{i}, u(t, x)\right) \leq\left\|\partial_{u} f\right\|_{\mathbf{L}^{\infty}\left([0, t] \times \mathcal{U}_{t} ; \mathbb{R}\right)}\left|u_{n}^{i}(t, x)-u(t, x)\right| .
$$

Hence, in the limit $n \rightarrow+\infty$ we have

$$
\lim _{n \rightarrow+\infty}[4.50]=\int_{0}^{T} \int_{\mathbb{R}_{+}} \Phi_{k}^{ \pm}(t, u(t, x)) \partial_{x} \varphi(t, x) \mathrm{d} x \mathrm{~d} t .
$$

Pass to (4.51):

$$
\begin{aligned}
& -\int_{\mathbb{R}_{+}} \eta_{k}^{ \pm}\left(u_{n}(T, x)\right) \varphi(T, x) \mathrm{d} x \\
= & -\int_{\mathbb{R}_{+}} \eta_{k}^{ \pm}(u(T, x)) \varphi(T, x) \mathrm{d} x+\int_{\mathbb{R}_{+}}\left(\eta_{k}^{ \pm}(u(T, x))-\eta_{k}^{ \pm}\left(u_{n}(T, x)\right)\right) \varphi(T, x) \mathrm{d} x \\
\leq & -\int_{\mathbb{R}_{+}} \eta_{k}^{ \pm}(u(T, x)) \varphi(T, x) \mathrm{d} x+\int_{\mathbb{R}_{+}}\left|u(T, x)-u_{n}(T, x)\right| \varphi(T, x) \mathrm{d} x,
\end{aligned}
$$


and the second term vanishes as $n \rightarrow+\infty$.

Concerning (4.52), we immediately get

$$
[4.52]] \leq\left\|\partial_{u} f\right\|_{\mathbf{L}^{\infty}([0, T] \times \mathcal{U} ; \mathbb{R})} \int_{0}^{T} \eta_{k}^{ \pm}\left(u_{b}(t)\right) \varphi(t, 0) \mathrm{d} t .
$$

We thus proved that $u$ solves 1.1 in the sense of Definition 2.1.

N.5) Lipschitz continuity in time. Consider $t_{1}, t_{2} \in[0, T]$, with $t_{1}<t_{2}$. Assume first that there exists $i \in\left\{0, \ldots, 2^{n}-1\right\}$ such that $t_{1}, t_{2} \in\left[T_{n}^{i}, T_{n}^{i+1}\right]$. Call $\mathcal{U}_{2}=\mathcal{U}_{t_{2}}=\mathcal{U}\left(u_{o}, u_{b \mid\left[0, t_{2}\right]}\right)$. Exploiting the wave front tracking approximation, compute

$$
\begin{aligned}
& \left\|u_{n}^{i}\left(t_{1}\right)-u_{n}^{i}\left(t_{2}\right)\right\|_{\mathbf{L}^{1}\left(\mathbb{R}_{+} ; \mathbb{R}\right)} \\
\leq & \left\|u_{n}^{i}\left(t_{1}\right)-u_{n}^{i, \varepsilon}\left(t_{1}\right)\right\|_{\mathbf{L}^{1}\left(\mathbb{R}_{+} ; \mathbb{R}\right)}+\left\|u_{n}^{i, \varepsilon}\left(t_{1}\right)-u_{n}^{i, \varepsilon}\left(t_{2}\right)\right\|_{\mathbf{L}^{1}\left(\mathbb{R}_{+} ; \mathbb{R}\right)}+\left\|u_{n}^{i, \varepsilon}\left(t_{2}\right)-u_{n}^{i}\left(t_{2}\right)\right\|_{\mathbf{L}^{1}\left(\mathbb{R}_{+} ; \mathbb{R}\right)} .
\end{aligned}
$$

The first and the third term converge to 0 as $\varepsilon \rightarrow 0$. To estimate the term in the middle, apply Formula (4.16) and exploit 4.40):

$$
\begin{aligned}
& \left\|u_{n}^{i, \varepsilon}\left(t_{1}\right)-u_{n}^{i, \varepsilon}\left(t_{2}\right)\right\|_{\mathbf{L}^{1}\left(\mathbb{R}_{+} ; \mathbb{R}\right)} \\
\leq & \left\|\partial_{u} f^{\varepsilon}\left(T_{n}^{i}\right)\right\|_{\mathbf{L}^{\infty}\left(\mathcal{U}_{2} ; \mathbb{R}\right)}\left(t_{2}-t_{1}\right) \\
& \times\left(\operatorname{TV}\left(u_{n}^{i-1, \varepsilon}\left(T_{n}^{i}\right)\right)+\operatorname{TV}\left(u_{b}^{\varepsilon} ;\left[T_{n}^{i}, t_{2}\right]\right)+\left|u_{b}^{\varepsilon}\left(T_{n}^{i}+\right)-u_{n}^{i-1, \varepsilon}\left(T_{n}^{i}, 0+\right)\right|\right) \\
\leq & \left\|\partial_{u} f\right\|_{\mathbf{L}^{\infty}\left(\left[0, t_{2}\right] \times \mathcal{U}_{2} ; \mathbb{R}\right)}\left(t_{2}-t_{1}\right)\left(V_{n}^{i, \varepsilon}-\operatorname{TV}\left(u_{b}^{\varepsilon} ;\left[t_{2}, T\right]\right)\right) \\
\leq & \cdots \\
\leq & \left\|\partial_{u} f\right\|_{\mathbf{L}^{\infty}\left(\left[0, t_{2}\right] \times \mathcal{U}_{2} ; \mathbb{R}\right)}\left(t_{2}-t_{1}\right)\left(\operatorname{TV}\left(u_{o}^{\varepsilon}\right)+\operatorname{TV}\left(u_{b}^{\varepsilon} ;\left[0, t_{2}\right]\right)+\left|u_{b}^{\varepsilon}(0+)-u_{o}^{\varepsilon}(0+)\right|\right) \\
\leq & C\left(t_{2}-t_{1}\right)
\end{aligned}
$$

where

$$
C=\left\|\partial_{u} f\right\|_{\mathbf{L}^{\infty}\left(\left[0, t_{2}\right] \times \mathcal{U}_{2} ; \mathbb{R}\right)}\left(\mathrm{TV}\left(u_{o}\right)+\mathrm{TV}\left(u_{b} ;\left[0, t_{2}\right]\right)+\left|u_{b}(0+)-u_{o}(0+)\right|\right) .
$$

Assume now that there exist $i, j \in\left\{0, \ldots, 2^{n}-1\right\}$, with $i<j$, such that $t_{1} \in\left[T_{n}^{i}, T_{n}^{i+1}\right]$ and $t_{2} \in\left[T_{n}^{j}, T_{n}^{j+1}\right]$. Therefore, exploiting the previous computation, we have

$$
\begin{aligned}
& \left\|u_{n}\left(t_{1}\right)-u_{n}\left(t_{2}\right)\right\|_{\mathbf{L}^{1}\left(\mathbb{R}_{+} ; \mathbb{R}\right)} \\
\leq & \left\|u_{n}^{i}\left(t_{1}\right)-u_{n}^{i}\left(T_{n}^{i+1}\right)\right\|_{\mathbf{L}^{1}\left(\mathbb{R}_{+} ; \mathbb{R}\right)}+\sum_{k=i+1}^{j-1}\left\|u_{n}^{k}\left(T_{n}^{k}\right)-u_{n}^{k}\left(T_{n}^{k+1}\right)\right\|_{\mathbf{L}^{1}\left(\mathbb{R}_{+} ; \mathbb{R}\right)}+\left\|u_{n}^{j}\left(T_{n}^{j}\right)-u_{n}^{j}\left(t_{2}\right)\right\|_{\mathbf{L}^{1}\left(\mathbb{R}_{+} ; \mathbb{R}\right)} \\
\leq & C\left(T_{n}^{i+1}-t_{1}\right)+\sum_{k=i+1}^{j-1} C\left(T_{n}^{k+1}-T_{n}^{k}\right)+C\left(t_{2}-T_{n}^{j}\right) \\
= & C\left(t_{2}-t_{1}\right),
\end{aligned}
$$

with $C$ as in 4.53). Let now $n$ tend to $+\infty$ : we obtain $\left\|u\left(t_{1}\right)-u\left(t_{2}\right)\right\|_{\mathbf{L}^{1}\left(\mathbb{R}_{+} ; \mathbb{R}\right)} \leq C\left(t_{2}-t_{1}\right)$, completing the proof of Point 2. 
N.6) Total Variation Estimate. Thanks to the lower semicontinuity of the total variation and to Point 4. in Proposition 2.3, we obtain the proof of of Point 3.:

$$
\operatorname{TV}(u(t)) \leq \lim _{n \rightarrow+\infty} \operatorname{TV}\left(u_{n}(t)\right) \leq \operatorname{TV}\left(u_{o}\right)+\operatorname{TV}\left(u_{b} ;[0, t]\right)+\left|u_{b}(0+)-u_{o}(0+)\right| .
$$

Proof of Theorem 2.6. Let $u_{n}$ and $v_{n}$ be defined as in (4.38), so that for $i=0, \ldots, 2^{n}-1$, $u_{n}^{i}$ and $v_{n}^{i}$ solve the autonomous IBVPs

$$
\left\{\begin{array}{lrl}
\partial_{t} u_{n}^{i}+\partial_{x} f\left(T_{n}^{i}, u_{n}^{i}\right)=0 & (t, x) \in\left[T_{n}^{i}, T_{n}^{i+1}\right] \times \mathbb{R}_{+} \\
u_{n}^{i}\left(T_{n}^{i}, x\right)=u_{n}^{i-1}\left(T_{n}^{i}, x\right) & x \in \mathbb{R}_{+} \\
u_{n}^{i}(t, 0)=u_{b}(t) & t \in\left[T_{n}^{i}, T_{n}^{i+1}\right]
\end{array}\right.
$$

and

$$
\left\{\begin{array}{lrl}
\partial_{t} v_{n}^{i}+\partial_{x} g\left(T_{n}^{i}, v_{n}^{i}\right)=0 & (t, x) \in\left[T_{n}^{i}, T_{n}^{i+1}\right] \times \mathbb{R}_{+} \\
v_{n}^{i}\left(T_{n}^{i}, x\right)=v_{n}^{i-1}\left(T_{n}^{i}, x\right) & x \in \mathbb{R}_{+} \\
v_{n}^{i}(t, 0)=u_{b}(t) & t \in\left[T_{n}^{i}, T_{n}^{i+1}\right] .
\end{array}\right.
$$

As in the proof of Proposition 2.5, for $i=0, \ldots, 2^{n}-1$ let $u_{n}^{i, \varepsilon}$ and $v_{n}^{i, \varepsilon}$ be the corresponding wave front tracking solutions. Observe that, for all $t \in[0, T]$,

$$
\|u(t)-v(t)\|_{\mathbf{L}^{1}\left(\mathbb{R}_{+} ; \mathbb{R}\right)}=\lim _{n \rightarrow+\infty}\left\|u_{n}(t)-v_{n}(t)\right\|_{\mathbf{L}^{1}\left(\mathbb{R}_{+} ; \mathbb{R}\right)} .
$$

Focus on the right hand side of (4.54). There exists $i \in\left\{0, \ldots, 2^{n}-1\right\}$ such that $t \in\left[T_{n}^{i}, T_{n}^{i+1}\right]$. Therefore,

$$
\begin{aligned}
& \left\|u_{n}(t)-v_{n}(t)\right\|_{\mathbf{L}^{1}\left(\mathbb{R}_{+} ; \mathbb{R}\right)} \\
= & \left\|u_{n}^{i}(t)-v_{n}^{i}(t)\right\|_{\mathbf{L}^{1}\left(\mathbb{R}_{+} ; \mathbb{R}\right)}+\left\|u_{n}^{i, \varepsilon}(t)-v_{n}^{i, \varepsilon}(t)\right\|_{\mathbf{L}^{1}\left(\mathbb{R}_{+} ; \mathbb{R}\right)}+\left\|v_{n}^{i, \varepsilon}(t)-v_{n}^{i}(t)\right\|_{\mathbf{L}^{1}\left(\mathbb{R}_{+} ; \mathbb{R}\right)} \\
\leq & \left\|u_{n}^{i}(t)-u_{n}^{i, \varepsilon}(t)\right\|_{\mathbf{L}^{1}\left(\mathbb{R}_{+} ; \mathbb{R}\right)}
\end{aligned}
$$

The first and the third term in 4.55 converge to 0 as $\varepsilon \rightarrow 0$, while an application of Proposition 2.2 and of Formula (4.33) allows to estimate the second term:

$$
\begin{aligned}
& \left\|u_{n}^{i, \varepsilon}(t)-v_{n}^{i, \varepsilon}(t)\right\|_{\mathbf{L}^{1}\left(\mathbb{R}_{+} ; \mathbb{R}\right)} \\
\leq & \left\|u_{n}^{i-1, \varepsilon}\left(T_{n}^{i}\right)-v_{n}^{i-1, \varepsilon}\left(T_{n}^{i}\right)\right\|_{\mathbf{L}^{1}\left(\mathbb{R}_{+} ; \mathbb{R}\right)} \\
& +\max \left\{1,\left\|\partial_{u} g\right\|_{\mathbf{L}^{\infty}([0, t] \times \mathcal{U} ; \mathbb{R})}\right\}\left\|\partial_{u}(f-g)\right\|_{\mathbf{L}^{\infty}([0, t] \times \mathcal{U} ; \mathbb{R})} \\
& \times\left(\operatorname{TV}\left(v_{n}^{i-1, \varepsilon}\left(T_{n}^{i}\right)\right)+\mathrm{TV}\left(u_{b}^{\varepsilon} ;\left[T_{n}^{i}, t\right]\right)+\left|u_{b}^{\varepsilon}\left(T_{n}^{i}+\right)-v_{n}^{i-1, \varepsilon}\left(T_{n}^{i}, 0+\right)\right|\right)\left(t-T_{n}^{i}\right),
\end{aligned}
$$

where $\mathcal{U}=\mathcal{U}\left(u_{o}, u_{b \mid[0, t]}\right)$ as in $(2.2)$, thanks to 4.8 and 4.48). Observe that the first term in 4.57) can be estimated by 4.40:

$$
\mathrm{TV}\left(v_{n}^{i-1, \varepsilon}\left(T_{n}^{i}\right)\right)+\operatorname{TV}\left(u_{b}^{\varepsilon} ;\left[T_{n}^{i}, t\right]\right)+\left|u_{b}^{\varepsilon}\left(T_{n}^{i}+\right)-v_{n}^{i-1, \varepsilon}\left(T_{n}^{i}, 0+\right)\right|
$$




$$
\begin{aligned}
& =V_{n}^{i-1, \varepsilon}-\operatorname{TV}\left(u_{b}^{\varepsilon} ;[t, T]\right) \\
& \leq \cdots \\
& \leq \operatorname{TV}\left(u_{o}\right)+\operatorname{TV}\left(u_{b} ;[0, t]\right)+\left|u_{b}(0+)-u_{o}(0+)\right|
\end{aligned}
$$

where in the last step we exploit (4.8). Concerning (4.56), we proceed recursively:

$$
\begin{aligned}
& {[4.56)] } \\
\leq & \left\|u_{n}^{i-2, \varepsilon}\left(T_{n}^{i-1}\right)-v_{n}^{i-2, \varepsilon}\left(T_{n}^{i-1}\right)\right\|_{\mathbf{L}^{1}\left(\mathbb{R}_{+} ; \mathbb{R}\right)} \\
& +\max \left\{1,\left\|\partial_{u} g\right\|_{\mathbf{L}^{\infty}([0, t] \times \mathcal{U} ; \mathbb{R})}\right\}\left\|\partial_{u}(f-g)\right\|_{\mathbf{L}^{\infty}([0, t] \times \mathcal{U} ; \mathbb{R})} \\
& \times\left[\operatorname{TV}\left(v_{n}^{i-2, \varepsilon}\left(T_{n}^{i-1}\right)\right)+\operatorname{TV}\left(u_{b}^{\varepsilon} ;\left[T_{n}^{i-1}, T_{n}^{i}\right]\right)+\left|u_{b}^{\varepsilon}\left(T_{n}^{i-1}+\right)-v_{n}^{i-2, \varepsilon}\left(T_{n}^{i-1}, 0+\right)\right|\right]\left(T_{n}^{i}-T_{n}^{i-1}\right) \\
\leq & \left\|u_{n}^{i-2, \varepsilon}\left(T_{n}^{i-1}\right)-v_{n}^{i-2, \varepsilon}\left(T_{n}^{i-1}\right)\right\|_{\mathbf{L}^{1}\left(\mathbb{R}_{+} ; \mathbb{R}\right)} \\
& +\max \left\{1,\left\|\partial_{u} g\right\|_{\mathbf{L}^{\infty}([0, t] \times \mathcal{U} ; \mathbb{R})}\right\}\left\|\partial_{u}(f-g)\right\|_{\mathbf{L}^{\infty}([0, t] \times \mathcal{U} ; \mathbb{R})} \\
& \times\left(\operatorname{TV}\left(u_{o}\right)+\operatorname{TV}\left(u_{b} ;[0, t]\right)+\left|u_{b}(0+)-u_{o}(0+)\right|\right)\left(T_{n}^{i}-T_{n}^{i-1}\right) .
\end{aligned}
$$

Therefore, thanks to 4.58 and 4.59), we obtain the estimate of 4.56-4.57):

$$
\begin{aligned}
\left\|u_{n}^{i, \varepsilon}(t)-v_{n}^{i, \varepsilon}(t)\right\|_{\mathbf{L}^{1}\left(\mathbb{R}_{+} ; \mathbb{R}\right)} \leq & \max \left\{1,\left\|\partial_{u} g\right\|_{\mathbf{L}^{\infty}([0, t] \times \mathcal{U} ; \mathbb{R})}\right\}\left\|\partial_{u}(f-g)\right\|_{\mathbf{L}^{\infty}([0, t] \times \mathcal{U} ; \mathbb{R})} \\
& \times\left(\operatorname{TV}\left(u_{o}\right)+\operatorname{TV}\left(u_{b} ;[0, t]\right)+\left|u_{b}(0+)-u_{o}(0+)\right|\right) t .
\end{aligned}
$$

Inserting (4.60) in (4.55) and letting $\varepsilon \rightarrow 0$, together with (4.54), concludes the proof.

Remark 4.6. If $T=+\infty$ : the above constructions can be completed on any time interval $[0, T]$. Thus, for any $T, T^{\prime}$, we obtain two maps $u_{T}$ and $u_{T^{\prime}}$ such that $u_{T^{\prime}}(t)=u_{T}(t)$ for $t \in\left[0, \min \left\{T, T^{\prime}\right\}\right]$, by Proposition 2.2 , and the above procedures can be extended to $t \in \mathbb{R}_{+}$.

Acknowledgment: The present work was supported by the PRIN 2012 project Nonlinear Hyperbolic Partial Differential Equations, Dispersive and Transport Equations: Theoretical and Applicative Aspects; by the INDAM-GNAMPA 2015 project Balance Laws in the Modeling of Physical, Biological and Industrial Processes and by the MATHTECH project funded by CNR-INDAM.

\section{References}

[1] D. Amadori and R. M. Colombo. Continuous dependence for $2 \times 2$ conservation laws with boundary. J. Differential Equations, 138(2):229-266, 1997.

[2] K. Ammar, P. Wittbold, and J. Carrillo. Scalar conservation laws with general boundary condition and continuous flux function. J. Differential Equations, 228(1):111-139, 2006.

[3] F. Ancona and A. Marson. Scalar non-linear conservation laws with integrable boundary data. Nonlinear Anal., 35(6, Ser. A: Theory Methods):687-710, 1999.

[4] C. Bardos, A. Y. le Roux, and J.-C. Nédélec. First order quasilinear equations with boundary conditions. Comm. Partial Differential Equations, 4(9):1017-1034, 1979. 
[5] S. Bianchini and R. M. Colombo. On the stability of the Standard Riemann Semigroup. Proc. Amer. Math. Soc., 130(7):1961-1973 (electronic), 2002.

[6] A. Bressan. Hyperbolic systems of conservation laws, volume 20 of Oxford Lecture Series in Mathematics and its Applications. Oxford University Press, Oxford, 2000. The one-dimensional Cauchy problem.

[7] R. M. Colombo, M. Mercier, and M. D. Rosini. Stability and total variation estimates on general scalar balance laws. Commun. Math. Sci., 7(1):37-65, 2009.

[8] R. M. Colombo and E. Rossi. Rigorous estimates on balance laws in bounded domains. Acta Math. Sci. Ser. B Engl. Ed., 35(4):906-944, 2015.

[9] C. M. Dafermos. Hyperbolic conservation laws in continuum physics, volume 325 of Grundlehren der Mathematischen Wissenschaften [Fundamental Principles of Mathematical Sciences]. Springer-Verlag, Berlin, third edition, 2010.

[10] F. Dubois and P. LeFloch. Boundary conditions for nonlinear hyperbolic systems of conservation laws. J. Differential Equations, 71(1):93-122, 1988.

[11] L. C. Evans and R. F. Gariepy. Measure theory and fine properties of functions. Studies in Advanced Mathematics. CRC Press, Boca Raton, FL, 1992.

[12] J. Goodman. Initial Boundary Value Problems for Hyperbolic Systems of Conservation Laws. $\mathrm{PhD}$ thesis, California University, 1982.

[13] H. Holden and N. H. Risebro. Front tracking for hyperbolic conservation laws, volume 152 of Applied Mathematical Sciences. Springer, New York, 2011. First softcover corrected printing of the 2002 original.

[14] S. N. Kružkov. First order quasilinear equations with several independent variables. Mat. Sb. (N.S.), 81 (123):228-255, 1970.

[15] J. Málek, J. Nečas, M. Rokyta, and M. Růžička. Weak and measure-valued solutions to evolutionary PDEs, volume 13 of Applied Mathematics and Mathematical Computation. Chapman \& Hall, London, 1996.

[16] S. Martin. First order quasilinear equations with boundary conditions in the $L^{\infty}$ framework. $J$. Differential Equations, 236(2):375-406, 2007.

[17] F. Otto. Ein Randwertproblem für skalare Erhaltugssätze. PhD thesis, Universität Bonn, 1993.

[18] F. Otto. Initial-boundary value problem for a scalar conservation law. C. R. Acad. Sci. Paris Sér. I Math., 322(8):729-734, 1996.

[19] D. Serre. Systems of conservation laws. 2. Cambridge University Press, Cambridge, 2000. Geometric structures, oscillations, and initial-boundary value problems, Translated from the 1996 French original by I. N. Sneddon.

[20] J. Vovelle. Convergence of finite volume monotone schemes for scalar conservation laws on bounded domains. Numer. Math., 90(3):563-596, 2002. 